\title{
On Hierarchical Routing in Doubling Metrics
}

\author{
T.-H. HUBERT CHAN, The University of Hong Kong \\ ANUPAM GUPTA, Carnegie Mellon University \\ BRUCE M. MAGGS, Duke University and Akamai Technologies \\ SHUHENG ZHOU, University of Michigan
}

\begin{abstract}
We study the problem of routing in doubling metrics and show how to perform hierarchical routing in such metrics with small stretch and compact routing tables (i.e., with a small amount of routing information stored at each vertex). We say that a metric $(X, d)$ has doubling dimension $\operatorname{dim}(X)$ at most $\alpha$ if every ball can be covered by $2^{\alpha}$ balls of half its radius. (A doubling metric is one whose doubling $\operatorname{dimension} \operatorname{dim}(X)$ is a constant.) We consider the metric space induced by the shortest-path distance in an underlying undirected graph $G$. We show how to perform $(1+\tau)$-stretch routing on such a metric for any $0<\tau \leq 1$ with routing tables of size at most $(\alpha / \tau)^{O(\alpha)} \log \Delta \log \delta$ bits with only $(\alpha / \tau)^{O(\alpha)} \log \Delta$ entries, where $\Delta$ is the diameter of the graph, and $\delta$ is the maximum degree of the graph $G$; hence, the number of routing table entries is just $\tau^{-O(1)} \log \Delta$ for doubling metrics. These results extend and improve on those of Talwar (2004).

We also give better constructions of sparse spanners for doubling metrics than those obtained from the routing tables earlier; for $\tau>0$, we give algorithms to construct $(1+\tau)$-stretch spanners for a metric $(X, d)$ with maximum degree at most $(2+1 / \tau)^{O(\operatorname{dim}(X))}$, matching the results of Das et al. for Euclidean metrics.
\end{abstract}

Categories and Subject Descriptors: F.2.2 [Analysis of Algorithms and Problem Complexity]: Nonnumerical Algorithms and Problems-Geometrical problems and computations; Computations on discrete structures

General Terms: Algorithms, Theory

Additional Key Words and Phrases: Doubling metrics, hierarchical routing, spanners

ACM Reference Format:

T.-H. Hubert Chan, Anupam Gupta, Bruce M. Maggs, and Shuheng Zhou. 2016. On hierarchical routing in doubling metrics. ACM Trans. Algorithms 12, 4, Article 55 (August 2016), 22 pages.

DOI: http://dx.doi.org/10.1145/2915183

A conference version of the article appeared in SODA 2005. The authors are alphabetically listed. This work was done when the authors were at Carnegie Mellon University, and was part of the first and the last authors' $\mathrm{PhD}$ theses.

Authors' addresses: T.-H. Hubert Chan, Department of Computer Science, the University of Hong Kong, Pokfulam Road, Hong Kong; email: hubert@cs.hku.hk; A. Gupta, Computer Science Department, Carnegie Mellon University, Pittsburgh PA 15213, USA; email: anupamg@cs.hku.hk; B. M. Maggs, Department of Computer Science, Duke University, Durham, NC 27708-0129, USA; email: bmm@cs.duke.edu. Supported in part by NSF Award CNF-0435382, NSF Award CNF-0433540, NSF ITR Award ANI-0331653, NSF ITR Award CCR-0205523, and US ARO Award DAAD19-02-1-0389. S. Zhou, Department of Statistics, University of Michigan, Ann Arbor, MI 48109, USA; email: shuhengz@umich.edu. We thank the members and companies of the PDL Consortium (including EMC, Engenio, Hewlett-Packard, HGST, Hitachi, IBM, Intel, Microsoft, Network Appliance, Oracle, Panasas, Seagate, Sun, and Veritas) for their interest, insights, feedback, and support. This material was based on research sponsored in part by the Army Research Office, under agreement number DAAD19-02-10389.

Permission to make digital or hard copies of part or all of this work for personal or classroom use is granted without fee provided that copies are not made or distributed for profit or commercial advantage and that copies show this notice on the first page or initial screen of a display along with the full citation. Copyrights for components of this work owned by others than ACM must be honored. Abstracting with credit is permitted. To copy otherwise, to republish, to post on servers, to redistribute to lists, or to use any component of this work in other works requires prior specific permission and/or a fee. Permissions may be requested from Publications Dept., ACM, Inc., 2 Penn Plaza, Suite 701, New York, NY 10121-0701 USA, fax +1 (212) 869-0481, or permissions@acm.org.

(c) 2016 ACM 1549-6325/2016/08-ART55 $\$ 15.00$

DOI: http://dx.doi.org/10.1145/2915183 


\section{INTRODUCTION}

The doubling dimension of a metric space $(X, d)$ is the least value $\alpha$ such that each ball of radius $R$ can be covered by at most $2^{\alpha}$ balls of radius $R / 2$ [Gupta et al. 2003]. For any $\alpha \in \mathbb{Z}$, the space $\mathbb{R}^{\alpha}$ under any of the $\ell_{p}$ norms has doubling dimension $\Theta(\alpha)$, and hence this doubling dimension extends the standard notion of geometric dimension; moreover, it can be seen as a way to parameterize the inherent "complexity" of metrics. In this article, we consider the metric space induced by the shortest-path distance in some underlying undirected graph $G$.

The conference version of this article was one of the first papers that started the design of routing algorithms for networks whose structure is parameterized by the doubling dimension $\operatorname{dim}(X)=\alpha$; our work has inspired a series of papers detailed in Section 1.2. We show that one can route along paths with stretch $(1+\tau)$ with small routing tables-with only $\left(\frac{\alpha}{\tau}\right)^{O(\alpha)} \log \Delta$ entries, where $\Delta$ is the diameter of the network. Each entry stores at most $O(\log \delta)$ bits, where $\delta$ is the maximum degree of the underlying graph. Hence, for doubling metrics-where $\alpha$ is a constant-and any $\tau \leq 1$, we have $(1+\tau)$-stretch routing with only $O(\log \Delta \log \delta)$ bits of routing information at each node.

The idea of placing restrictions on the growth rate of networks to bound their "intrinsic complexity" has been around for a long time (see, e.g., Kleinrock and Kamoun [1977]) and has recently been used in several contexts in the literature on object location in peer-to-peer networks [Plaxton et al. 1999; Karger and Ruhl 2002; Hildrum et al. 2002]. While these papers used definitions and restrictions that differ slightly from each other, we note that our results hold in those models as well. Our results improve those of Talwar [2004], whose routing schemes for metrics with $\operatorname{dim}(X)=\alpha$ require local routing information of $\approx O\left(\log ^{\alpha} \Delta\right)$ bits. Formally, we have the following main result.

Theorem 1.1. Given any network $G$ inducing a metric $(X, d)$ with $\operatorname{dim}(X)=\alpha$ and any $\tau>0$, there is a routing scheme on $G$ that achieves $(1+\tau)$-stretch and where each node stores only $\left(\frac{\alpha}{\tau}\right)^{O(\alpha)} \log \Delta \log \delta$ bits of routing information.

The proof of the theorem proceeds along familiar lines; we construct a set of hierarchical decompositions (HDs) of the metric $(X, d)$, where each HD consists of a set of successively finer partitions of $X$ with geometrically decreasing diameters. Each node in $X$ maintains a table containing next hops to a small subset of clusters in these partitions; to route a packet from $s$ to $t$, we use the routing table for $s$ to pick some "small cluster" $C$ in $s$ 's table that contains $t$ and send the packet to some node $x$ in $C$; a similar process repeats at node $x \in C$ until the packet reaches $t$. The idea is to create routing tables that ensure that the distance from $x$ to $t$ is much smaller than that from $s$ to $t$, and hence the detour taken in going from $s$ to $t$ (via intermediate points such as $x$ ) is only $\tau d(s, t)$. (Details of routing schemes appear in Section 4.)

Our construction is novel in the following sense: the standard ways to construct HDs are top-down methods that iteratively refine partitions while we use a bottom-up approach. The reason is that top-down methods create long-range dependencies that require us to build $O(\log n) \mathrm{HDs}$ in general; in order to use the locality of the doubling metrics and get away with $\tilde{O}(\alpha)$ HDs, we develop a bottom-up approach that avoids these dependencies when building HDs. The analysis of this process uses the Lovász Local Lemma (in a way similar to Krauthgamer and Lee [2003] and Gupta et al. [2003]); the details are given in Section 3.

Apart from the aforementioned result on low-stretch routing, the proof of Theorem 1.1 can be used to infer the existence of linear-sized spanners for doubling metrics, that is, subgraphs with only $O_{\tau, \alpha}(n)$ edges that maintain distances to be within a factor 
of $(1+\tau)$. We further give simpler and tighter constructions of spanners, extending similar results of Das et al. [1995] for Euclidean metrics.

THEOREM 1.2. Every metric $(X, d)$ has a $(1+\tau)$-spanner $H$ where the degree of each vertex is at most $(2+1 / \tau)^{O(\operatorname{dim}(X))}$; hence, $H$ has a linear number of edges for any constant $\tau$ and $\operatorname{dim}(X)$.

\subsection{Related Work}

One of the papers that influenced this work is that of Kleinrock and Kamoun [1977]. They describe a general hierarchical clustering model on which our routing schemes are based. They show that routing schemes based on a hierarchical clustering model do not cause much increase in the average path length for networks that satisfy the following two assumptions: (1) the diameter of any cluster $S$ chosen is bounded above by $O\left(|S|^{v}\right)$ for some constant $v \in[0,1]$, and (2) the average distance between nodes in the network is $\Theta\left(n^{\nu}\right)$. In contrast, we give bounds on the path stretch on a per node-pair level using slightly different assumptions on the network geometry.

Distributed packet routing protocols have been widely studied in the theoretical computer science community; see, for example, Frederickson and Janardan [1988, 1989], Awerbuch and Peleg [1992], Peleg and Upfal [1989], Cowen [2001], and Peleg [2000] or the survey by Gavoille [2001] on some of the issues and techniques. Note that these results, however, are usually for general networks or for networks with some topological structure. By placing restrictions on the doubling dimension, we are able to give results that degrade gracefully as the "complexity" of the metric increases. For example, it is known that any universal routing algorithm with stretch less than 3 requires some node to store at least $\Omega(n)$ routing information [Gavoille and Gengler 2001]; however, these graphs generate metrics with large $\operatorname{dim}(X)$. Our results thus allow one to circumvent these lower bounds for metrics of "lower dimension."

Packet routing in low-dimensional networks has been previously studied in Talwar [2004], which gives algorithms that require $O\left(\alpha\left(\frac{6}{\tau \alpha}\right)^{\alpha}\left(\log ^{\alpha+2} \Delta\right)\right)$ bits of information to be stored per node in order to achieve $(1+\tau)$-stretch routing-for constant stretch $\tau$ and doubling dimension $\alpha$. For (small) constant dimension $\alpha, \log \Delta=\Omega(\log n)$. The resulting dependence on $O\left(\log ^{\alpha+2} \Delta\right)$ of Talwar's scheme should be contrasted with the dependence of $O(\log \Delta \log \delta)$ bits of information in our schemes. However, for large doubling dimension, Talwar's scheme can perform better than ours. We should point out that his algorithms are based on graph decomposition ideas with a top-down approach and do not require the Lovász Local Lemma to construct routing tables.

Other papers on object location in peer-to-peer networks [Plaxton et al. 1999; Karger and Ruhl 2002; Hildrum et al. 2002] have also used restrictions similar to Kleinrock and Kamoun [1977] on the growth rate of metrics; in particular, they consider metrics where increasing the radius of any ball by a factor of 2 causes the number of points in it to increase by at most some constant factor $2^{\beta}$. (Plaxton et al. [1999] also consider the lower bound on the growth.) Here the parameter $\beta$ can be considered to be another notion of "dimension" for a metric space. It can be shown that $\operatorname{dim}(X) \leq 4 \beta$ [Gupta et al. 2003, Proposition 1.2]; hence, our results hold for such metrics as well. Our scheme is also similar in spirit to a data-tracking scheme of Rajaraman et al. [2001], who use approximations by tree distributions to obtain bounds on the stretch incurred.

Finally, sparse spanners have been studied widely, having found applications in network algorithms (see, e.g., Peleg [2000]), since they allow us to store information about the metric compactly. Our work extends a series of results on Euclidean spanners with bounded degree by Vaidya [1991], Salowe [1992], Arya et al. [1995], and Das et al. [1995]. Independent of our work, Gao et al. [2004] gave a similar sparse spanner 
construction based on nets for Euclidean space, but they did not optimize the spanner degree, which can have a logarithmic dependence on the metric diameter. Har-Peled and Mendel [2005] have also obtained, among many other results, constructions of sparse spanners for doubling metrics; they also give nearly linear-time procedures to find these constructions.

\subsection{Later Development}

Since the conference version of this article appeared, there has been much follow-up work on compact routing in networks with a bounded doubling dimension. In particular, Slivkins [2005] has improved the routing information stored at each node to $\left(\frac{1}{\tau}\right)^{O(\alpha)} \log \Delta \log \delta$ bits. One might wonder why our routing table size has a factor $\left(\frac{\alpha}{\tau}\right)^{O(\alpha)}$. The reason is that since we would like to apply the Lovász Local Lemma to our padded probabilistic hierarchical decompositions, we need to construct the partitions in a bottom-up manner. Since a cluster must not be divided in its parent level, in order to satisfy the padding property, one way to resolve this is to require the distance scales between successive levels to be at least a factor $\alpha$ apart. Therefore, each cluster could have $\alpha^{O(\alpha)}$ child clusters, and this is why we have a worse bound. In retrospect, one could have directly used hierarchical nets (with constant factor increase in distance scales) to construct a routing scheme, as demonstrated by Slivkins, without the use of probabilistic decomposition. However, the use of the Lovász Local Lemma in our article has inspired subsequent research in local embeddings [Abraham et al. 2007] and low-dimensional embeddings [Chan et al. 2010] for doubling metrics. Moreover, Moser and Tardos [2010] gave a constructive proof of the general Lovász Local Lemma, which can simplify our algorithm in Section 3.2.

Observe that the diameter $\Delta$ can be exponentially large, and actually for doubling metrics, one can show $\log \Delta=\Omega(\log n)$. Abraham et al. [2006] and Konjevod et al. [2007] have further removed the dependence on the diameter to obtain $\left(\frac{1}{\tau}\right)^{O(\alpha)} \log ^{3} n$ bits of information per node.

We work under the model where the header of a routing packet contains the information of the target node, and in particular the protocol designer can choose labels for the nodes. There has been work under the model of name-independent routing [Awerbuch et al. 1990; Awerbuch and Peleg 1990; Abraham et al. 2006; Konjevod et al. 2007], in which the packet header can contain only the predetermined identity of a node, which the protocol designer cannot modify.

There has also been follow-up work on spanners for doubling metrics. Chan and Gupta [2006] considered hop diameters and gave a construction of $(1+\tau)$-spanners with $n \tau^{-O(\alpha)}$ edges that achieves a hop diameter that grows like the functional inverse of the Ackermann's function. They also provided a construction of $(1+\tau)$-spanners with nearly a linear number of edges (i.e., only $n \log ^{*} n+n \tau^{-O(\alpha)}$ edges) and a constant hop diameter. Gottlieb and Roditty [2008a, 2008b] showed constructions of fully dynamic spanners for doubling metrics. Gottlieb and Roditty [2008b] gave a construction of $(1+\tau)$-spanners with $O(n)$ edges of constant degree that supports updates in $O(\log n)$ worst-case time (taking $\tau$ and $\alpha$ as constant), which is optimal up to a constant even for insertion-only operations.

In the past decade, there has been a series of work on the tradeoffs between various spanner parameters that has eventually led to the breakthrough result by Elkin and Solomon [2013], who gave a spanner construction for doubling metrics that has constant degree, logarithmic hop diameter, and logarithmic lightness compared to minimum spanning tree. The result has been generalized to fault-tolerant spanners by Chan et al. [2015] and further improved by Solomon [2014]. The reader can refer to the 
references of these recent papers for a more comprehensive description of the latest development.

\section{DEFINITIONS AND NOTATION}

Let the input metric be $(X, d)$; this article deals with finite metrics with at least two points. We use standard terminology from the theory of metric spaces; many definitions can be found in Deza and Laurent [1997] and Heinonen [2001]. Given $x \in X$ and $r \geq 0$, we let $\mathbf{B}(x, r)$ denote $\left\{x^{\prime} \in X \mid d\left(x, x^{\prime}\right) \leq r\right\}$, that is, the ball of radius $r$ around $x$. Given a subset $S \subseteq X$, the distance of $x \in X$ to the set $S$ is $d(x, S)=\min \left\{d\left(x, x^{\prime}\right) \mid x^{\prime} \in S\right\}$.

The doubling constant $\lambda_{X}$ of a metric space $(X, d)$ is the smallest value $\lambda$ such that every ball in $X$ can be covered by $\lambda$ balls of half the radius. The doubling dimension of $X$ is then defined as $\operatorname{dim}(X)=\log _{2} \lambda_{X}$; we use the letter $\alpha$ to denote $\operatorname{dim}(X)$. A metric is called doubling when its doubling dimension is a constant. A subset $Y \subseteq X$ is an $r$-net of $X$ if (1) for every $x, y \in Y, d(x, y) \geq r$ and (2) $X \subseteq \cup_{y \in Y} \mathbf{B}(y, r)$. Such nets always exist for any $r>0$ and can be found using a greedy algorithm.

Proposition 2.1 (See, E.G., Gupta Et AL. [2003]). If all pairwise distances in a set $Y \subseteq X$ are at least $r$ (e.g., when $Y$ is an $r$-net of $X)$, then for any point $x \in X$ and radius $t$, we have that $|\mathbf{B}(x, t) \cap Y| \leq \lambda_{X}^{\left\lceil\log _{2} \frac{2 t}{r}\right\rceil}$.

A cluster $C$ in the metric $(X, d)$ is just a subset of points of the set $X$. The diameter of the cluster $C$ is the largest distance between points of the cluster. Each cluster is associated with a center $x \in X$ (which may not lie in $C$ ) and the radius of the cluster $C$ is the smallest value $r$ such that the cluster $C$ is contained in $\mathbf{B}(x, r)$.

Definition 2.2. Given $r>0$, an $\boldsymbol{r}$-ball partition $\Pi$ of $(X, d)$ is a partition of $X$ into clusters $C_{1}, C_{2}, \ldots$, with each cluster $C_{i}$ having a radius at most $r$.

By scaling, let us assume that the smallest interpoint distance in $X$ is exactly 1 . Let $\Delta$ denote the diameter of the metric $(X, d)$, and hence $\Delta$ is also the aspect ratio of the metric. Define $\rho=256 \alpha+1$ and $h=\left\lceil\log _{\rho} \Delta\right\rceil$. Let us define $\eta_{i}=1+\rho+\rho^{2}+\cdots+\rho^{i}<$ $\rho^{i+1} /(\rho-1)$; note that $\eta_{i}=\rho \eta_{i-1}+1$. Let us fix a $\rho^{i} / 2$-net and denote with $N_{i}$ for the metric $(X, d)$, for every $0 \leq i \leq h+1$.

\subsection{Hierarchical Decompositions}

We now give a formal definition of a hierarchical decomposition (HD), which is used throughout this article and is the basic object of our study. As noted later, such a decomposition can be naturally associated with a decomposition tree that is used for our hierarchical routing schemes.

Definition 2.3. A $\rho$-hierarchical decomposition $\Pi(\rho-H D)$ of the metric $(X, d)$ is a sequence of partitions $\Pi_{0}, \ldots, \Pi_{h}$ with $h=\left\lceil\log _{\rho} \Delta\right\rceil$ such that:

(1) The partition $\Pi_{h}$ has one cluster $X$, the entire set.

(2) (geometrically decreasing diameters) The partition $\Pi_{i}$ is an $\eta_{i}$-ball partition. Since interpoint distances are at least 1 , it implies that $\Pi_{0}=\{\{x\}: x \in X\}$; in other words, each cluster in $\Pi_{0}$ is a singleton vertex.

(3) (hierarchical) $\Pi_{i}$ is a refinement of $\Pi_{i+1}$ and each cluster in $\Pi_{i}$ is contained within some cluster of $\Pi_{i+1}$.

Given such a $\rho$-HD $\Pi=\left(\Pi_{i}\right)_{i=0}^{h}$, the partition $\Pi_{i}$ is called the level-i partition of $\Pi$ and clusters in $\Pi_{i}$ are the level-i clusters. Note that these clusters have a radius $\eta_{i}$ and hence diameter $\leq 2 \eta_{i}$. Furthermore, define the degree $\operatorname{deg}(\Pi)$ to be the maximum number of level- $i$ clusters contained in any level- $(i+1)$ cluster in $\Pi_{i+1}$, for all $0 \leq i \leq h-1$. 
2.1.1. Hierarchical Decompositions and HSTs. A hierarchical decomposition is a laminar family of sets, where given any two sets, either they are disjoint or one contains the other. It is well known that such a family $\mathcal{F}$ of sets over $X$ can be associated with a natural decomposition tree whose vertices are sets in $\mathcal{F}$ and whose leaves are all the smallest sets in the family (which are elements of $X$, in this case). We can use this to associate a so-called hierarchically well-separated tree (also called an HST [Bartal 1996]) $T_{\Pi}$ with a hierarchical decomposition $\Pi$; since each edge in $T_{\Pi}$ connects some $C \in \Pi_{i}$ and $C^{\prime} \in \Pi_{i-1}$ with $C^{\prime} \subseteq C$, we associate a length $\eta_{i}$ with edge $\left(C, C^{\prime}\right)$. Given such a tree $T_{\Pi}$, we can (and indeed do) talk about its level- $i$ clusters with no ambiguity; these are the same level- $i$ clusters in the associated $\Pi_{i}$. Note that the degree of vertices in this tree $T_{\Pi}$ is bounded by $\operatorname{deg}(\Pi)+1$.

\subsection{Padded Probabilistic Ball Partitions}

Recall that an $r$-ball partition $\Pi$ of $(X, d)$ is a partition of $X$ into a set of clusters $C \subseteq X$, each contained in a ball $\mathbf{B}(v, r)$ for some $v \in X . \mathbf{B}(x, t)$ is cut in the partition $\Pi$ if there is no cluster $C \in \Pi$ such that $\mathbf{B}(x, t) \subseteq C$. In general, $\mathbf{B}(x, t)$ is cut by a set $S \subseteq X$ if both $S \cap \mathbf{B}(x, t)$ and $\mathbf{B}(x, t) \backslash S$ are nonempty.

Let $\mathcal{P}$ be a collection of all possible partitions of $X$, and hence $\Pi \in \mathcal{P}$. Given a partition $\Pi \in \mathcal{P}$ and $x \in X$, let $C_{\Pi}(x)$ be the cluster of $\Pi$ containing $x$.

Definition 2.4 (Gupta et al. [2003]). An $(r, \varepsilon)$-padded probabilistic ball partition of a metric $(X, d)$ is a probability distribution $\mu$ over $\mathcal{P}$ satisfying:

(1) (bounded radius) Each $\Pi$ in the support of $\mu$ is an $r$-ball partition.

(2) (padding) $\forall x \in X, \operatorname{Pr}_{\mu}\left[d\left(x, X \backslash C_{\Pi}(x)\right) \geq \varepsilon r\right] \geq \frac{1}{2}$.

(This is called a padded probabilistic decomposition in Gupta et al. [2003].) Each cluster $C$ in every partition $\Pi$ in the support of a probabilistic ball partition $\mu$ has radius at most $r$; and for any $x \in X$, a random $r$-ball partition $\Pi$ drawn from the distribution $\mu$ does not cut $\mathbf{B}(x, \varepsilon r)$ (and hence $\mathbf{B}(x, \varepsilon r)$ is contained in cluster $C_{\Pi}(x) \in \Pi$ ) with probability $\geq 1 / 2$.

\section{PADDED PROBABILISTIC HIERARCHICAL DECOMPOSITIONS}

In this section, we define a $(\rho, \varepsilon)$-padded probabilistic hierarchical decomposition (PPHD) of the metric $(X, d)$, on which the routing algorithm is based. A PPHD is a probability distribution over HDs that has a "probabilistic padding" property similar to that in Definition 2.4. For any pair of nodes $s, t$ in $X$ and any ball containing both $s$ and $t$ with a diameter of $\approx d(s, t)$, the PPHD ensures that this ball is contained in a single cluster of radius only slightly $(\approx \alpha$ factor $)$ larger than $d(s, t)$ at a suitable level with probability $\geq \frac{1}{2}$. Thus, the shortest $s$ - $t$ path is contained entirely in this cluster of radius not much more than $d(s, t)$. This is the general intuition for PPHDs and the starting point for the routing algorithm.

For our applications, we refine PPHDs so that they consist of only $m=O(\alpha \log \alpha)$ HDs. In Section 3.1, we first give an existence proof, using the Lovász Local Lemma (LLL), to show that such decompositions exist. In Section 3.2, we then outline a randomized polynomial-time algorithm to find the decompositions using Beck's techniques [Beck 1991].

The existence proof for the PPHDs has the following outline. We first give a randomized algorithm to form a single random hierarchical decomposition $\Pi$, which proves the existence of PPHDs, albeit with support over an exponential number of HDs. To reduce the size to something that depends only on $\alpha$, we have to use the locality property of the metric space and the LLL. One significant complication in the proof is that we cannot use the standard top-down decomposition schemes to construct PPHDs, since they have 
long-range correlations that preclude the application of the LLL. Our solution to this problem is to build the decomposition trees in a bottom-up fashion and to make sure that the coarser partitions respect the cluster boundaries made in the finer partitions.

\subsection{Existence of PPHDs}

Motivated by the routing application, we are interested in finding the following structure, which we call a $(\rho, \varepsilon)$-padded probabilistic hierarchical decomposition. This is a probability distribution $\mu$ over $\rho$-hierarchical decompositions (as defined in Definition 2.3) so that given $\mathbf{B}(x, \varepsilon r)$ with $r \approx \rho^{i}$, if we choose a random $\rho$-HD $\Pi$ from $\mu$ and examine the partition $\Pi_{i}$ in it, $\mathbf{B}(x, \epsilon r)$ is cut in this partition $\Pi_{i}$ with probability at most $\frac{1}{2}$.

Definition 3.1 (PPHD). A $(\rho, \varepsilon)$-padded probabilistic hierarchical decomposition (referred to as a $(\rho, \varepsilon)$-PPHD) is a distribution $\mu$ over $\rho$-hierarchical decompositions, such that for any point $x \in X$ and any value $r$ s.t. $\rho^{i-1} \leq r \leq \rho^{i}$,

$$
\underset{\Pi \in \mu}{\operatorname{Pr}}\left[\mathbf{B}(x, \varepsilon r) \text { is cut in } \Pi_{i}\right] \leq \frac{1}{2},
$$

where the random $\rho$-hierarchical decomposition chosen is $\Pi=\left(\Pi_{i}\right)_{i=0}^{h}$. The degree of the PPHD $\mu$ is defined to be $\operatorname{deg}(\mu)=\max _{\Pi \in \mu} \operatorname{deg}(\Pi)$.

Note that the definition of a PPHD extends both the idea of a padded probabilistic ball partition and that of HDs-we ask for a distribution over entire HDs, instead of over ball partitions at a certain scale $r$. However, having picked a random $\rho$-HD $\Pi=\left(\Pi_{i}\right)_{i=0}^{h}$ from this distribution, we demand that balls of radius $\approx \varepsilon \rho^{i}$ be cut with small probability only in partition $\Pi_{i}$ that is "at the correct distance scale." Our main theorem of this section is the following:

Theorem 3.2. Given a metric $(X, d)$, there exists a $(\rho, \varepsilon)$-PPHD $\mu$ for $(X, d)$ with $\rho=O(\alpha)$ and $\varepsilon=O(1 / \alpha)$. The degree $\operatorname{deg}(\mu)$ of the PPHD is at most $\alpha^{O(\alpha)}$. Furthermore, there exists a distribution with the aforementioned properties $\mu_{m}$ whose support is over only $m=O(\alpha \log \alpha) H D$ s.

Since any hierarchical decomposition $\Pi$ can be associated with a tree $T_{\Pi}$ (as mentioned in Section 2.1), the previous theorem can be viewed as guaranteeing a set of $m$ trees such that the level- $i$ clusters in half of these trees do not cut a given ball of radius $\approx \varepsilon \rho^{i}$. This proves the existence of an appropriate tree cover.

Definition 3.3. A stretch- $k$ Steiner tree cover for $(X, d)$ is a set of trees $\mathcal{T}=\left\{T_{1}, \ldots, T_{m}\right\}$ (with each tree $T_{i}$ possibly containing Steiner points $\notin X$, and edges having lengths), where for every $x, x^{\prime} \in X$, there exists a tree $T_{i} \in \mathcal{T}$ for $(X, d)$ such that the (unique shortest) path in $T_{i}$ between $x$ and $x^{\prime}$ has length at most $k d\left(x, x^{\prime}\right)$.

Lemma 3.4. Given a metric $(X, d)$ with $\operatorname{dim}(X)=\alpha$, there exists a stretch- $O(\rho / \varepsilon)$ Steiner tree cover consisting of $O(\alpha \log \alpha)$ trees, where each tree has degree at most $\alpha^{O(\alpha)}$.

We omit the simple proof of the previous lemma and the description of how the Steiner points can be removed from the trees without altering distances and degrees. We prove Theorem 3.2 in the rest of this section. We first prove (in Section 3.1.1) that one can obtain the result where the PPHD $\mu$ has support over many HDs. We then use the Lovász Local Lemma (in Section 3.1.2) to show that a PPHD distribution $\mu_{m}$ with support over only a small number of HDs exists.

3.1.1. Padded Probabilistic Hierarchical Partitions. If we do not care about the number of HDs in the support of a PPHD, the existence result of Theorem 3.2 has been proved 
0 . Let $Y \leftarrow X, p \leftarrow \frac{c \alpha \Gamma}{\Lambda}$ for constant $c$ to be fixed later,

$N$ be a $\Lambda / 2$-net of $X$.

1. Pick an arbitrary "root" vertex $v \in N$ not picked before

2. Set the initial value of the "radius" $L \leftarrow \Lambda / 2$

3. Flip a coin with bias $p$

4. If the coin comes up heads, goto Step 11

5. If the coin comes up tails, increment $L$ by $\Gamma$

6. If $L>\Lambda(1-1 / 4 \alpha)$

7. $\quad$ choose a value $\hat{L}$ from $[0, \Lambda /(4 \alpha)]$ u.a.r.

8. round down $\hat{L}$ to the nearest multiple of $\Gamma$

9. $\quad$ set $L \leftarrow \Lambda(1-1 / 4 \alpha)+\hat{L}$

10. Else goto Step 3

11. Form a new cluster $C^{\prime}$ in $\Pi^{\prime \prime}$ containing all clusters in $\Pi^{\prime} \cap Y$ with centers liying in $\mathbf{B}(v, L)$

12. Remove the vertices in $C^{\prime}$ from $Y$

13. (Remark: $C^{\prime}$ has radius at most $\Lambda+\Gamma$ )

14. If $Y \neq \emptyset$ goto Step 1

15. End

Fig. 1. Algorithm Cut-Clusters.

earlier [Talwar 2004] with better guarantees; the proof basically follows from the padded decompositions given in Gupta et al. [2003]. However, we now give another proof that introduces ideas that are ultimately useful in obtaining a PPHD distribution whose support is over a small number of HDs.

Theorem 3.5. Given a metric $(X, d)$, there exists a $(\rho, \varepsilon)$-PPHD $\mu$ for $(X, d)$ with $\rho=O(\alpha)$ and $\varepsilon=O(1 / \alpha)$, and with degree $\operatorname{deg}(\mu)=\alpha^{O(\alpha)}$. Furthermore, one can sample from $\mu$ in polynomial time.

Proof. We define a randomized process that builds a random hierarchical decomposition tree in a bottom-up fashion, instead of the usual top-down way. To build an HD $\Pi$, we start with $\left(\Pi_{0}=\{\{x\}: x \in X\}\right)$ and perform an inductive step. At any step, we are given a partial structure $\left(\Pi_{i}, \ldots, \Pi_{0}\right)$, where for each $j \leq i$, the clusters in $\Pi_{j-1}$ (which is an $\eta_{j-1}$-ball partition) are contained within the clusters of $\Pi_{j}$. We then build a new partition $\Pi_{i+1}$, with all clusters of $\Pi_{i}$ being contained within clusters of $\Pi_{i+1}$. We have to ensure that clusters of $\Pi_{i+1}$ are contained in balls of radius at most $\eta_{i+1}$ and that any ball of radius $\varepsilon r$ for $\rho^{i} \leq r \leq \rho^{i+1}$ is cut in $\Pi_{i+1}$ with probability at most $\frac{1}{2}$. This way, we end up with a valid random HD $\Pi$. The claimed probability distribution $\mu$ is the one naturally generated by this algorithm. To create the clusters of $\Pi_{i+1}$, we use a decomposition procedure whose property is summarized in the following lemma.

Lemma 3.6. Given a metric $(X, d)$ with a $\Gamma$-ball partition $\Pi^{\prime}$ of $X$ into clusters lying in balls of radius at most $\Gamma \geq 1$, and a value $\Lambda \geq 8 \Gamma$, there is a randomized algorithm to create a $(\Lambda+\Gamma)$-ball partition $\Pi^{\prime \prime}$ of $X$, where each cluster of $\Pi^{\prime}$ is contained in some cluster of $\Pi^{\prime \prime}$, and for any $x \in X$ and radius $0 \leq r \leq \Lambda$,

$$
\operatorname{Pr}\left[\mathbf{B}(x, r) \text { is cut in } \Pi^{\prime \prime}\right] \leq \frac{O(r+\Gamma)}{\Lambda} \alpha .
$$

Proof. Note that we can assume that $\Gamma<\Lambda / c \alpha$ and $\Lambda \geq \alpha$, since otherwise the lemma is trivially true. Using the algorithm CuT-CLusters given in Figure 1, we create 
a partition of $Y$ (and hence of $X$ ); all distances are measured according to the original distance function $d$ in $X$.

Let us define $\mathcal{B}_{x}=\mathbf{B}(x, r)$. Note that if $\mathcal{B}_{x}$ is cut in $\Pi^{\prime \prime}$ due to some value of $L$ from $v \in N$ (for the first time), then $L$ falls into the interval $[d(v, x)-r-\Gamma, d(v, x)+r+\Gamma]$. Indeed, if $\mathcal{B}_{x}$ is cut in $\Pi^{\prime \prime}$, there are at least two clusters $C_{1}^{\prime}, C_{2}^{\prime} \in \Pi^{\prime}$ such that they both cut $\mathcal{B}_{x}$, and $\mathbf{B}(v, L)$ contains one of their centers but not both. Since both clusters intersect $\mathcal{B}_{x}$, their centers $c_{1}^{\prime}$ and $c_{2}^{\prime}$ are at distance at most $r+\Gamma$ from $x$. If $L<d(v, x)-r-\Gamma$, the triangle inequality implies that $\mathbf{B}(v, L)$ cannot contain either center. Similarly, if $L>d(v, x)+r+\Gamma, \mathbf{B}(v, L)$ contains both of them. Hence, the value of $L$ must fall into the interval indicated earlier.

If a cut in Steps 11 and 12 is made due to the appearance of a heads in Step 4, we call such a cut a normal cut; otherwise, we call it a forced cut. We now bound the probability that the ball $\mathcal{B}_{x}=\mathbf{B}(x, r)$ is cut due to either type.

Normal cuts. Consider the first instant in time when the parameter $L$ for some root $v \in N$ reaches a value such that the cluster obtained by taking all $\Pi^{\prime} \cap Y$ clusters with centers in $B(v, L)$ would cut $\mathcal{B}_{x}$. (If there is no such time, then $\mathcal{B}_{x}$ is never cut by a normal cut.) In this case, $L$ must also be in the range $d(v, x) \pm(r+\Gamma)$, and increases with time. Now either (1) we make a normal cut before $L$ goes outside this range (2) we make a forced cut, or (3) $L$ goes outside the range and we make no cut in this range. In any case, the fate of $\mathcal{B}_{x}$ is decided; $\mathcal{B}_{x}$ is either cut or contained in a new cluster with center $v$. We now upper-bound the probability that event (1) happens. There are at most $2(r+\Gamma) / \Gamma$ coin flips made (with bias $p$ ) when the value of $L$ is in the correct range of width at most $2(r+\Gamma)$ and one of these flips must come up heads for the cut to be made. The trivial union bound now shows this probability to be at most $\frac{2(r+\Gamma)}{\Gamma} p=\frac{2 c(r+\Gamma)}{\Lambda} \alpha$.

Forced cuts. Let us look at some root $v \in N$ and bound the probability that a forced cut is made with cutting radius $L$ from $v$ in some range $\mathcal{R}_{x}=d(v, x) \pm(r+\Gamma)$. Since the cut is forced and the value of $L$ is greater than $\Lambda(1-1 / 4 \alpha) \geq 3 \Lambda / 4$, we must have flipped a sequence of at least $\Lambda / 4 \Gamma$ successive tails; the probability of this event is at most

$$
(1-p)^{(\Lambda / 4 \Gamma)} \leq e^{-p \Lambda / 4 \Gamma}=e^{-\frac{c}{4} \alpha} .
$$

Now, we choose $\hat{L}$ to be a multiple of $\Gamma$ uniformly in a range of width at most $\Lambda / 4 \alpha$, and hence the probability that $L$ falls into a range of length $2(r+\Gamma)$ is at most $2(r+\Gamma) /(\Lambda / 4 \alpha)$. Multiplying this by Equation (1), we obtain a bound of $e^{-\frac{c}{4} \alpha} \times \frac{8(r+\Gamma)}{\Lambda} \alpha$ on the probability that a forced cut is made around $v$ with $L$ in the range $\mathcal{R}_{x}$ such that the cluster $C^{\prime}$ with center $v$ in $\Pi^{\prime \prime}$ may cut $\mathcal{B}_{x}$. Finally, for any $x \in X, \mathcal{B}_{x}$ can only be cut by clusters from roots $v \in N$ that are at distance at most $(r+\Gamma)+\Lambda \leq 3 \Lambda$ from $x$; by Proposition 2.1, there are at most $|\mathbf{B}(x, 3 \Lambda) \cap N|=\left(\frac{6 \Lambda}{\Lambda / 2}\right)^{\alpha} \leq(12)^{\alpha}$ of such roots. Now we choose $c$ to be large enough; the probability of $B_{x}$ being forcedly cut due to any such root is at most $12^{\alpha} \times e^{-\frac{c}{4} \alpha} \times \frac{8(r+\Gamma)}{\Lambda} \alpha \leq \frac{O(r+\Gamma)}{\Lambda} \alpha$ by the union bound.

We now use the previous lemma to prove Theorem 3.5. Using $\Pi^{\prime}=\Pi_{i}, \Gamma=\eta_{i}<$ $\rho^{i}(\rho /(\rho-1))$, and $\Lambda=\eta_{i+1}-\Gamma=\rho^{i+1}$, and using $N=N_{i+1}\left(\right.$ which is a $\rho^{i+1} / 2=\Lambda / 2$ net), we create a $\left(\Gamma+\Lambda=\eta_{i+1}\right)$-ball partition such that for all $x$ and all $r \leq \rho^{i+1}$ and $\varepsilon=O(1 / \alpha)$, we have

$$
\operatorname{Pr}[\mathbf{B}(x, \varepsilon r) \text { cut }] \leq \frac{O(\varepsilon r+\Gamma)}{\Lambda} \alpha \leq \frac{O\left(\rho^{i}\right)}{\rho^{i+1}} \alpha \leq \frac{1}{10}<\frac{1}{2},
$$

for $\rho / \alpha$ and $c$ being large enough constants. The probability distribution $\mu$ over all decompositions $\Pi$ thus generated satisfies the requirements of a PPHD as given in 
Definition 3.1. Finally, we bound the degree $\operatorname{deg}(\mu)$ of the PPHD $\mu$; note that each level- $i$ cluster is centered at some $v \in N_{i}$; hence, the number of level-i clusters contained in some level- $(i+1)$ cluster is $\left(2 \eta_{i+1} /\left(\rho^{i} / 2\right)\right)^{O(\alpha)}=\alpha^{O(\alpha)}$ by Proposition 2.1.

Few Hierarchical Decompositions. The previous proof immediately gives us a PPHD $\mu_{M}$ with a support on only $M=O(\log n+\log \log \Delta)$ HDs. By sampling from the distribution $\mu$ for $M$ times, we get the HDs $\Pi^{(1)}, \ldots, \Pi^{(M)}$, and let the PPHD $\mu_{M}$ be the uniform distribution on these HDs. By Equation (2), for each $j \in[1 \ldots M]$, point $x \in X$, and radius $r \leq \rho^{i}, \mathbf{B}(x, \varepsilon r)$ is not cut in the partition $\Pi_{i}^{(j)}$ with probability $1 / 10$; hence, a Chernoff bound implies that this ball is cut in the level-i partitions of more than $M / 2$ of the HDs with probability less than $1 /(n \log \Delta)^{O(1)}$. Now, taking the trivial union bound over all possible values of the center $x \in X$ and all the log $\Delta$ values of $r$ that are powers of 2 shows that the $\mu_{M}$ is a $(\rho, \varepsilon / 2)$-PPHD whp.

3.1.2. Even Fewer Hierarchical Decompositions. While the proof of Theorem 3.5 and the discussion earlier do not produce a PPHD with small support (of size $O(\alpha \log \alpha)$ ), we have seen all the essential ideas required to prove the existence of such a distribution $\mu_{m}$ and hence to complete the proof of Theorem 3.2. To prove this result, we use the locality of the construction in conjunction with the LLL. This locality property is the very reason we built the hierarchical decomposition bottom-up; it ensures that if any particular ball is not cut at some low level $i$ (the "local decisions"), it is not cut at levels higher than $i$ (i.e., the "nonlocal decisions"). Also, we choose the decomposition procedure of Theorem 3.6 in preference to others (e.g., those in Gupta et al. [2003] and Talwar [2004]) since they choose a single random radius for all clusters in one particular partition $\Pi$ of $X$, which causes correlations across the entire metric space. (The LLL has been used in similar contexts in Gupta et al. [2003] and Krauthgamer and Lee [2003].)

Proof of Theorem 3.2. To show that there is a distribution $\mu_{m}$ over only $m=$ $O(\alpha \log \alpha)$ trees, we use an idea similar to that in the previous section, augmented with some ideas from Gupta et al. [2003]. Instead of building one hierarchical decomposition $\Pi$ bottom-up, we build $m$ hierarchical decompositions $\Pi^{(1)}, \ldots, \Pi^{(m)}$ simultaneously (also from the bottom up).

As before, the proof proceeds inductively; we assume that we are given level- $i$ partitions $\Pi_{i}^{(1)}, \ldots, \Pi_{i}^{(m)}$, where $\Pi_{i}^{(j)}$ is the level-i partition belonging to $\Pi^{(j)}$. We then show that we can build level- $(i+1)$ partitions $\Pi_{i+1}^{(1)}, \ldots, \Pi_{i+1}^{(m)}$ where each $\Pi_{i}^{(j)}$ is a refinement of the corresponding $\Pi_{i+1}^{(j)}$, and any given ball $\mathbf{B}(x, \varepsilon r)$ with $\rho^{i} \leq r \leq \rho^{i+1}$ is cut in at most $m / 2$ of these level- $(i+1)$ partitions. We start off this process with each $\Pi_{0}^{(j)}=\{\{x\}: x \in X\}$ being the partition consisting of all singleton points in $X$. Let $J=\{1, \ldots, m\}$. Given $m$ level-i partitions $\left(\Pi_{i}^{(j)}\right)_{j \in J}$, we create $m$ level- $(i+1)$ partitions $\left(\Pi_{i+1}^{(j)}\right)_{j \in J}$ using the procedure in Lemma 3.6 independently on each of the $m$ decompositions; parameters are set as in the proof of Theorem 3.5, with $\Lambda=\rho^{i+1}, \Gamma=\eta_{i}$, and $\varepsilon=1 / O(\alpha)$. This extends the $m$ hierarchical decompositions to the $(i+1)^{s t}$ level; it remains to show that the probability of balls being cut is small.

To describe the events of interest, let us take $\beta=\varepsilon \rho^{i+1}$ and define $Z$ to be a $\beta$-net of $X$. For each $z \in Z$, define $\mathcal{B}_{z}$ to be $\mathbf{B}(z, 2 \beta)$, and $\mathcal{E}_{z}^{i+1}$ to be an event in which $\mathcal{B}_{z}$ is cut in more than $m / 2$ of the partitions $\left(\Pi_{i+1}^{(j)}\right)_{j=1}^{m}$, which we refer to as a "bad" event (used in Section 3.2). We prove the claim using the Lovász Local Lemma.

$$
\text { Claim 3.7. Given any }\left(\Pi_{i}^{(j)}\right)_{j=1}^{m}, \operatorname{Pr}\left[\bigwedge_{z \in Z} \overline{\mathcal{E}_{z}^{i+1}}\right]>0 \text {. }
$$


Lemma 3.8 (Lovász Local Lemma). Given a set of events $\left\{\mathcal{E}_{z}^{i+1}\right\}_{z \in Z}$, suppose that each event is mutually independent of all but at most B other events. Further suppose that, for each event $\mathcal{E}_{z}^{i+1}, \operatorname{Pr}\left[\mathcal{E}_{z}^{i+1}\right] \leq p$. Then, if $\operatorname{e} p(B+1)<1, \operatorname{Pr}\left[\bigwedge_{z \in Z} \overline{\mathcal{E}}^{i+1}\right]>0$.

Proof of Claim 3.7. First, let us calculate the probability of $\mathcal{E}_{z}^{i+1}$ : by changing the constant in $\varepsilon$, we can make the probability that a ball $\mathcal{B}_{z}$ is cut in one level- $(i+1)$ partition to be at most $1 / 8$. Let us denote by $A_{z}^{j}$ the event that $\mathcal{B}_{z}$ is cut in partition $\Pi_{i+1}^{(j)}$. The expected number of partitions in which the ball is cut is at most $m / 8$. Since the partitions are constructed independently, the probability for the event $\mathcal{E}_{z}^{i+1}$ that $\mathcal{B}_{z}$ is cut in $m / 2$ partitions (which is at least four times the expectation) is at most $\exp (-9 m / 40)$; this can be established using a standard Chernoff bound. This, in turn, is at most $(0.8)^{m}$, which we define to be $p$.

Next we show that an event $\mathcal{E}_{z}^{i+1}$ is mutually independent of all events $\mathcal{E}_{z^{\prime}}^{i+1}$ such that $d\left(z, z^{\prime}\right)>4 \eta_{i+1}$. For each partition $\Pi_{i+1}^{(j)}$, each root $v \in N_{i+1}$ determines its radius by conducting a random experiment independent of any other roots' experiments. These random experiments, and only these, determine whether events such as $A_{z}^{j}$ occur. In turn, whether event $\mathcal{E}_{z}^{i+1}$ occurs is determined only by events $A_{z}^{1}, \ldots, A_{z}^{m}$. For a particular $j$, for each $z$, all of the cuts that could affect $\mathcal{B}_{z}$ in the algorithm CuT-Clusters are made from roots $v \in N_{i+1}$ at distance at most $2 \beta+\Gamma+\Lambda=2 \beta+\eta_{i+1}<2 \eta_{i+1}$ from $z$. Whether event $A_{z}^{j}$ occurs is determined by the experiments corresponding to these roots alone. If $d\left(z, z^{\prime}\right)>4 \eta_{i+1}$, then there is no intersection between the experiments for $z$ and the experiments for $z^{\prime}$. Since $\mathcal{E}_{z}^{i+1}$ is determined by $A_{z}^{1}, \ldots, A_{z}^{m}, \mathcal{E}_{z}^{i+1}$ is mutually independent of the set of all $\mathcal{E}_{z^{\prime}}^{i+1}$ such that $d\left(z, z^{\prime}\right)>4 \eta_{i+1}$.

We apply the LLL now. Note that the number of $z^{\prime} \in Z$ within distance $4 \eta_{i+1}$ of $\mathcal{E}_{z}^{i+1}$ for $z \in Z$ is at most $\left|\mathbf{B}\left(z, 4 \eta_{i+1}\right) \cap Z\right| \leq\left(\frac{8 \eta_{i+1}}{\beta}\right)^{\alpha} \leq O(\alpha)^{\alpha}$. We define this quantity to be $B$; e $p(B+1)$ is at most 1 for $m=O(\alpha \log \alpha)$ and Claim 3.7 follows.

Having proved the claim, let us now show that with nonzero probability, each $\mathbf{B}(x, r)$ for $x \in X$ and $\rho^{i} \leq r \leq \rho^{i+1}$ is not cut in at least $m / 2$ of the level- $(i+1)$ partitions $\left(\Pi_{i+1}^{(j)}\right)_{j \in J}$. Let us call this event $S C_{i+1}$. The claim shows that with nonzero probability, each ball $\mathcal{B}_{z}$ with $z \in Z$ is not cut in at least $m / 2$ of the partitions $\left(\Pi_{i+1}^{(j)}\right)_{j \in J}$. Since each $x \in X$ is at distance at most $\beta$ to some $z_{x} \in Z$, the triangle inequality implies that $\mathbf{B}(x, \varepsilon r) \subseteq \mathbf{B}(x, \beta)$ is not cut if $\mathbf{B}\left(z_{x}, 2 \beta\right)$ is not cut, which holds in at least half of the partitions. Hence, $S C_{i+1}$ also holds with nonzero probability.

Finally, we prove that we can choose a random set of HDs $\left(\Pi^{(j)}\right)_{j \in J}$ such that $S C_{i+1}$ occurs for each $1 \leq i+1 \leq h$ simultaneously with nonzero probability. The key to the proof is that we have assumed an arbitrary (worst-case) set of partitions $\left(\Pi_{i}^{(j)}\right)_{j=1}^{m}$ at level $i$ in proving a nonzero lower bound on $\operatorname{Pr}\left[S C_{i+1}\right]$. Hence, we can ignore any dependence among the events $S C_{i+1}$ for $1 \leq i+1 \leq h$, and simply multiply their nonzero probabilities together to obtain a nonzero lower bound on the probability that they all occur simultaneously.

\subsection{An Algorithm for Finding the Decompositions}

The previous procedure can be made algorithmic using an approach based on Beck's algorithmic version of the LLL (see, e.g., Alon and Spencer [1992] and Beck [1991]). We also remark that one can apply the techniques of Moser and Tardos [2010] to achieve an algorithmic version of the LLL. The decomposition satisfies all properties of the one that is shown to exist using LLL in Theorem 3.2, although with some changes in 
constant parameter values. As in the proof of Theorem 3.2, we build $m=O(\alpha \log \alpha)$ HDs level by level in a bottom-up fashion.

On any particular level $i+1$, we begin by choosing $m$ partitions at random. After making the random choices, we examine the partitions and identify all of the bad events that have occurred. We then group together bad events that may depend on each other, as well as "good" events that may depend on the bad events. Each group forms a connected component in the LLL dependency graph. We show that, with high probability, all connected components have size $O(\log v)$, where $v=|Z|$ is the size of the $\varepsilon \rho^{i+1}$-net of $X$.

Once the groups have been identified, we need to eliminate the bad events. Hence, for each group, we "undo" all of the random choices concerning that group, while not modifying any choices that do not affect the group. New choices must be made for each group so that no bad event occurs. Because the group size is small (the number of centers $v \in N_{i+1}$ concerning the group that we choose random radius for is also $O(\log v)$ ), we can find new settings for these choices using exhaustive search in polynomial time.

One interesting complication in this proof is that the set of clusters containing a group have different shapes in the $m$ different partitions. In each partition, we cut out a "hole" and redo the choices within the hole. The boundary of the hole is formed from the boundaries of the clusters that may influence the bad events (and the good events) in the group. In forming the boundary, additional good events may be added to the hole. As a consequence, it is possible that a good event inside a hole in one partition may appear inside a different hole in another partition. Hence, when we perform exhaustive search, these holes must be considered together. However, our method of bounding the size of each connected component already takes into account any merging of holes on account of shared good events, so that we never have to redo the choices for a group of size more than $O(\log v)$.

Another issue is that the subset of centers in a hole that belong to $N_{i+1}$, the $\rho^{i+1} /$ 2 -net that covers the entire metric, may not by themselves cover the hole. (Portions of the hole may be covered by centers outside the hole.) So for each of the $m$ partitions, we may have to add additional net points inside the hole to obtain a complete cover for it. We show that the size of net points in the hole increases by only a constant factor and remains $O(\log v)$, and the degree of the hierarchical decomposition trees is at most $\alpha^{O(\alpha)}$ as before.

\section{THE $(1+\tau)$-STRETCH ROUTING SCHEMES}

Given a $(\rho, \varepsilon)$-PPHD $\mu_{m}$ with a support on $m$ HDs, we can now define, for every $0<\tau \leq 1$, a $(1+\tau)$-stretch routing scheme that uses routing tables of size at most $m(\alpha / \tau)^{\bar{O}(\alpha)} \log \Delta \log \delta$ bits at every node.

We consider a routing scheme in which each node can only forward packets to its direct neighbors in the underlying graph $G$. Formally, a routing scheme consists of the following components:

(1) Addressing Scheme. Given the underlying network $G$ (and the induced metric space), each point $x$ is assigned some global address $\operatorname{addr}(x)$.

(2) Routing Table. Each point $x$ stores partial information on the network in its local routing table Route $_{x}$.

(3) Packet Header Generation. Given the global addresses of the origin $s$ and the destination $t$, generate a packet header that contains routing information toward $t$. The packet header could be modified at intermediate points as the packet is sent from $s$ to $t$. 
(4) Forwarding Algorithm. Given a packet header, an intermediate point $x$ uses

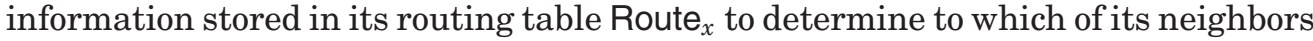
in $G$ to forward the packet. The packet header might also be modified.

Let us recall some of the notation defined earlier. Let $\left(\boldsymbol{\Pi}^{(j)}\right)_{j=1}^{m}$ be the $m$ hierarchical decompositions on which $\mu_{m}$ has positive support, and the level- $i$ partition corresponding to $\Pi^{(j)}$ be called $\Pi_{i}^{(j)}$. Recall that we can associate each hierarchical decomposition $\Pi^{(j)}$ with a tree $T_{j}$ (as outlined in Section 2.1). Note that each of these trees has a $\operatorname{deg}\left(\mu_{m}\right)$ bounded by $\alpha^{O(\alpha)}$ and a height of at most $h=\left\lceil\log _{\rho} \Delta\right\rceil$. Recall that each internal vertex of the tree $T_{j}$ at level $i$ corresponds to a cluster of $\Pi_{i}^{(j)}$ and leaves of $T_{j}, \forall j \in J$ correspond to vertices in $X$, where $J=\{1, \ldots, m\}$. Let each internal vertex $v$ of each tree $T_{j}$ label its children by numbers between 1 and $\operatorname{deg}\left(\mu_{m}\right) ; v$ does not label anything with the number 0 , but uses it to refer to its parent. Note that this allows us to represent any path in a tree $T_{j}$ by a sequence of at most $2 h=O\left(\log _{\rho} \Delta\right)$ labels.

Lemma 3.4 already shows that the $m$ trees thus created form a small $O(\rho / \varepsilon)=O\left(\alpha^{2}\right)$ stretch Steiner tree cover, which can be used for routing purposes (as in Section 4.3). However, since such a large stretch is not always acceptable, we improve on this scheme in the following subsections to get better routing bounds.

\subsection{The Addressing Scheme}

Given a tree $T_{j}$ and a vertex $x \in X$, we assign $x$ a local address $\operatorname{addr}_{j}(x)$, which consists of $h=\left\lceil\log _{\rho} \Delta\right\rceil$ blocks, one for each level of the tree $T_{j}$. Each block has a fixed length. The $i^{\text {th }}$ block of the $\operatorname{addr}_{j}(x)$ corresponds to partition $\Pi_{i}^{(j)}$ and contains the label assigned to the cluster $C_{x}$ containing $x$ in $\Pi_{i}^{(j)}$ by $C_{x}$ 's parent in $T_{j}$. Since any such label is just a number between 1 and $\operatorname{deg}\left(\mu_{m}\right)$, where $\operatorname{deg}\left(\mu_{m}\right)=\alpha^{O(\alpha)}$, we need $O(\alpha \log \alpha)$ bits per block. In fact, one can extend this addressing scheme to any cluster $C$ in $T_{j}$. If $C$ is a level-i cluster, the $k^{\text {th }}$ block of $\operatorname{addr}_{j}(C)$ contains $* \mathrm{~s}$ for $k<i$; $\operatorname{addr}_{j}(X)$ for the root cluster of $T_{j}$ contains all $*$ s matching all vertices in $X$.

The global address $\operatorname{addr}(x)$ of point $x \in X$ is the concatenation $\left\langle\operatorname{addr}_{1}(x), \ldots, \operatorname{addr}_{m}(x)\right\rangle$ of its local addresses $\operatorname{addr}_{j}(x)$ for $j \in J$. Since each cluster $C$ belongs to only one tree $T_{j}$, we define $\operatorname{addr}_{j^{\prime}}(C)$ to be a sequence of \#s of the correct length (where \#s are dummy symbols matching nothing), and hence define a global address of $C$ as well. (This is only for simplicity; in actual implementations, cluster addresses for $T_{j}$ can be given by the tuple $\left\langle\operatorname{addr}_{j}(C), j\right\rangle$.)

Since there are $O(\alpha \log \alpha)$ bits per block, $h$ blocks per local address, and $m$ local addresses per global address, substitution of the appropriate values gives the address length $A$ to be at most $m \times h \times\left\lceil\log \left(\operatorname{deg}\left(\mu_{m}\right)\right)\right\rceil=O(\alpha \log \alpha) \times\left\lceil\log _{\rho} \Delta\right\rceil \times O(\alpha \log \alpha)=$ $O\left(\alpha^{2} \log \alpha \log \Delta\right)$ bits.

\subsection{The Routing Table}

For each point $x \in X$, we maintain a routing table Route $_{x}$ that contains the following information for each $T_{j}, 1 \leq j \leq m$ :

(1) For each ancestor of $x$ in $T_{j}$ that corresponds to a cluster $C$ containing $x$, we maintain a table entry for $C$.

(2) Moreover, for each such $C$, we maintain an entry for each descendant of $C$ in $T_{j}$ reachable within $\ell$ hops in tree $T_{j}$. Here $\ell=\Theta\left(\log _{\rho} 1 / \varepsilon \tau\right)$, with the constants chosen such that $\eta_{i-\ell} \leq \frac{\varepsilon \tau}{4} \rho^{i-1}$. 
In the routing table Route for $_{x}$, each of the previous entries thus corresponds to some level- $i^{\prime}$ cluster $C^{\prime}$ in $T_{j}$. Let close ${ }_{x}\left(C^{\prime}\right)$ be the closest point in $C^{\prime}$ to $x$. (We assume, w.l.o.g., that ties are broken in some consistent way, so that any node $y$ on a shortest path from $x$ to close $_{x}\left(C^{\prime}\right)$ has the value close $y\left(C^{\prime}\right)=$ close $_{x}\left(C^{\prime}\right)$; in fact, this consistency is the only property we use.) For this $C^{\prime}$, Route $x$ stores (a) the global address $\operatorname{addr}\left(C^{\prime}\right)$ by which the table is indexed, (b) the identity of a "next hop" neighbor $y$ of $x$ that stays on a shortest path from $x$ to the closest point close ${ }_{x}\left(C^{\prime}\right)$ in $C^{\prime}$, and (c) an extra bit ValidPath ${ }_{x}\left(C^{\prime}\right)$ : if the cluster $\ell$ level above $C^{\prime}$ in $T_{j}$ is the cluster $C$, then $\operatorname{ValidPath}_{x}\left(C^{\prime}\right)$ is set to be true if $\mathbf{B}\left(x, \varepsilon \rho^{i^{\prime}+\ell}\right)$ is entirely contained within cluster $C$ and $d\left(x\right.$, close $\left._{x}\left(C^{\prime}\right)\right) \leq \varepsilon \rho^{i^{\prime}+\ell}$, and is set to be false otherwise. Of course, if we reach the root of $T_{j}$ while trying to go up $\ell$ levels, then the bit is set to be true.

Lemma 4.1. The number of entries in the routing table Route of $_{x}$ any $x \in X$ is at most $\log \Delta \times(\alpha / \tau)^{O(\alpha)}$.

PRoOF. Let us estimate the number of entries in Route for $_{\text {any }} x \in X$. There are $m$ trees. For each tree $T_{j}$, for all $j \in J$, there are $h=\left\lceil\log _{\rho} \Delta\right\rceil$ ancestors of $x$ and the degree of the tree is bounded by $\operatorname{deg}\left(\mu_{m}\right)=\alpha^{O(\alpha)}$. Recall that $\rho$ and $1 / \varepsilon$ are both $O(\alpha)$, and hence $\ell=O(\log (\alpha / \tau))$. Plugging these values in, we get that the number of entries for $x$ across $m$ trees is at most $m \times h \times\left(\operatorname{deg}\left(\mu_{m}\right)\right)^{\ell}=O(\alpha \log \alpha) \times O\left(\log _{\alpha} \Delta\right) \times \alpha^{O(\alpha \ell)}=\log \Delta \times(\alpha / \tau)^{O(\alpha)}$. Each entry is indexed by one global address (of at most $A=O\left(\alpha^{2} \log \alpha \log \Delta\right)$ bits, which we do not store in Route $_{x}$ since we can deduce it from $\operatorname{addr}(x)$ based on the clustering structure); each entry indeed contains the identity of the next hop (which uses $O(\log \delta)$ bits, where $\delta$ is the maximum degree of $G$ ), and one additional ValidPath bit.

The forwarding algorithm makes use of two functions, NextHop $x$ and PrefMatch . For a point $x$ and a level- $i^{\prime}$ cluster $C^{\prime}$ in $T_{j}$, the function NextHop $x\left(\operatorname{addr}\left(C^{\prime}\right)\right)$ returns the next hop on the path from $x$ to close $_{x}\left(C^{\prime}\right)$ provided that the next hop does not leave the cluster $C$ at level $i^{\prime}+\ell$ that contains $C^{\prime}$, and null otherwise. (As we shall see, the packet-forwarding algorithm is guaranteed never to encounter a null next hop.) Given points $x$ and $t$ in $X$, the function PrefMatch ${ }_{x}(t)$ returns an $\operatorname{addr}\left(C^{\prime}\right)$ in Route $_{x}$ such that in some $T_{j}, t$ belongs to the level-i cluster $C^{\prime}$, ValidPath ${ }_{x}\left(C^{\prime}\right)$ is true, and the value $i$ is the smallest across all trees. Note that both of these functions can be computed efficiently by node $x$. Furthermore, it is possible to support the functions with data structures of size comparable to that of Route . .

Note that once the points in $X$ have been assigned addresses (for which we have described only an offline algorithm), the routing tables can be built up in a completely distributed fashion. In particular, a distributed breadth-first-search algorithm can be applied to determine whether a ball of a certain radius is cut in a particular decomposition, and a distributed implementation of the Bellman-Ford algorithm can be used to establish the next-hop entries for destinations for which the shortest paths lie within a certain cluster.

\subsection{The Forwarding Algorithm}

The idea behind the forwarding algorithm is to start a packet off from its origin $s$ toward an intermediate cluster $C$ containing its destination $t$; the packet header thus consists of two pieces of information $\langle\operatorname{addr}(t), \operatorname{addr}(C)\rangle$, where $t$ is the destination node for the packet and $C$ is the intermediate cluster containing $t$. Initially, the cluster can be chosen (degenerately) to be the root cluster of (say) tree $T_{1}$.

Upon reaching a node $x$ in the intermediate cluster $C$, a new and smaller intermediate cluster $C^{\prime}$, also containing $t$, must be chosen, possibly from a different tree; the packet header must be updated with $\operatorname{addr}\left(C^{\prime}\right)$ that remains the same until reaching $C^{\prime}$. Suppose 
1. Let packet header be $\langle\operatorname{addr}(t), \operatorname{addr}(C)\rangle$.

2. If $C$ contains $x$, the current node, then

3. $\quad$ find $\operatorname{addr}\left(C^{\prime}\right) \leftarrow \operatorname{PrefMatch}_{x}(t)$

4. $\quad$ let $y \leftarrow \operatorname{NextHop}_{x}\left(\operatorname{addr}\left(C^{\prime}\right)\right)$

5. forward packet with new header $\left\langle\operatorname{addr}(t), \operatorname{addr}\left(C^{\prime}\right)\right\rangle$ to $y$.

6. Else (now $x \notin C$ )

7. $\quad$ let $y \leftarrow \operatorname{NextHop}_{x}(\operatorname{addr}(C))$

8. forward packet with unchanged header $\langle\operatorname{addr}(t), \operatorname{addr}(C)\rangle$ to $y$.

9. End

Fig. 2. The forwarding algorithm at node $x$.

that the new cluster $C^{\prime}$ containing $t$ is at level $i^{\prime}$. After selecting this cluster, the packet is sent off toward $C^{\prime}$ with the new header, following a shortest path that stays within the cluster $\hat{C}$ at level $i^{\prime}+\ell$ that contains both $x$ and $C^{\prime}$. This process is repeated until ultimately the packet reaches the cluster containing only the destination $t$. The algorithm is presented in Figure 2.

Theorem 4.2. The forwarding algorithm has a stretch of at most $(1+\tau)$, where $\tau \leq 1$.

Proof. We first show that the algorithm is indeed valid; each of the steps can be executed and the packet eventually reaches $t$. Suppose that the packet has just reached a node $x$ in an intermediate cluster $C$ containing $t$ (with $\operatorname{addr}(C)$ in its header); thus, $x$ needs to execute Step 3 to find a new cluster $C^{\prime}$ containing $t$. Clearly, PrefMatch ${ }_{x}(t)$ can return the root cluster $C_{\text {root }}$ of any $T_{j}$, since it contains $t$. We show, however, that the cluster $C^{\prime}$ returned by $\operatorname{PrefMatch}_{x}(t)$ has a small diameter and nodes along a valid shortest path from $x$ to $C^{\prime}$ will forward the packet correctly until it reaches $C^{\prime}$.

LEMMA 4.3. If the packet is at node $x$ with distance to the target $t$ being $d(x, t) \leq \varepsilon \rho^{i}$, Step 3 must return some addr $\left(C^{\prime}\right)$ such that cluster $C^{\prime} \ni t$ is at level $(i-\ell)$ or lower in some $T_{j^{\prime}}$ with ValidPath ${ }_{x}\left(C^{\prime}\right)$ being true. Furthermore, all of vertex $v$ on all shortest paths from $x$ to close ${ }_{x}\left(C^{\prime}\right)=$ close $_{v}\left(C^{\prime}\right)$ has a nonnull NextHop $\left(\operatorname{addr}\left(C^{\prime}\right)\right)$.

Proof. The $(\rho, \varepsilon)$-PPHD ensures that there exists at least one tree $T_{j}$ such that $\mathbf{B}\left(x, \varepsilon \rho^{i}\right)$ is not cut in the level-i partition $\Pi_{i}^{(j)}$; let $\hat{C}_{\text {cont }} \in \Pi_{i}^{(j)}$ be the level-i cluster in $T_{j}$ that contains $\mathbf{B}\left(x, \varepsilon \rho^{i}\right)$.

Let $C_{t} \in \Pi_{i-\ell}^{(j)}$ be the level- $(i-\ell)$ cluster in $T_{j}$ containing $t$. The $\operatorname{ValidPath}_{x}\left(C_{t}\right)$ bit must be true since $\mathbf{B}\left(x, \varepsilon \rho^{i}\right) \subseteq \hat{C}_{\text {cont }}$ in $\Pi_{i}^{(j)}$ and $d\left(x\right.$, close $\left.{ }_{x}\left(C_{t}\right)\right) \leq d(x, t) \leq \varepsilon \rho^{i}$; thus, PrefMatch $_{x}$ can (and may indeed) just return $\operatorname{addr}\left(C_{t}\right)$ given no "better" choices. However, PrefMatch ${ }_{x}$ always finds a cluster $C^{\prime}$ in some $T_{j^{\prime}}$ at the lowest level across all trees, such that $t \in C^{\prime}$, and ValidPath ${ }_{x}\left(C^{\prime}\right)$ is true in Route ${ }_{x}$. Let the level of $C^{\prime}$ be $i^{\prime}$; the value $i^{\prime}$ is at most $(i-\ell)$. Now let $\hat{C} \in \Pi_{i^{\prime}+\ell}^{\left(j^{\prime}\right)}$ be the cluster $\ell$ levels above $C^{\prime} \in \Pi_{i^{\prime}}^{\left(j^{\prime}\right)}$ in $T_{j^{\prime}}$ that contains both $x$ and $C^{\prime}$. (Such $\hat{C}$ must exist at level $i^{\prime}+\ell$ for $\operatorname{addr}\left(C^{\prime}\right)$ to be in

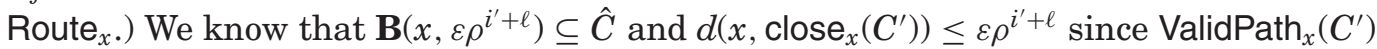

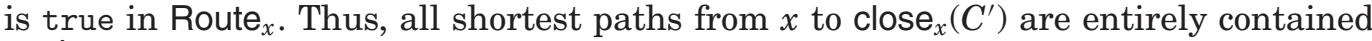
in $\hat{C}$. Hence, the $\operatorname{NextHop}_{v}\left(\operatorname{addr}\left(C^{\prime}\right)\right)$ pointer at any node $v$ on one of these paths must be nonnull since all shortest paths from $v$ to close $_{v}\left(C^{\prime}\right)=\operatorname{close}_{x}\left(C^{\prime}\right)$ are all contained in $\hat{C}$, the cluster $\ell$ levels above $C^{\prime}$ in $T_{j}^{\prime}$.

It remains to bound the path stretch. Consider the case when a packet is sent from $s$ to $t$. Let $C^{\prime}$ be a cluster at level $i-\ell$ returned by Step 3 of the forwarding algorithm. 
Note that if the level $i \leq \ell$, then $C^{\prime}=\{t\}$, and we send the packet directly to $t$ with $\tau=0$. Using these short distances as the base case, we now do induction on the distance from $s$ to $t$.

If $C^{\prime}$ is a nontrivial cluster containing $t$, then we go on a shortest path from $s$ to some vertex $v=$ close $_{s}\left(C^{\prime}\right) \in C^{\prime}$. Since $t \in C^{\prime}, d(s, v) \leq d(s, t)$. Because the diameter of $C^{\prime}$ is at most $2 \eta_{i-\ell}, d(v, t) \leq 2 \eta_{i-\ell}<\varepsilon \rho^{i-1}<d(s, t)$. (The last inequality holds because if $\varepsilon \rho^{i-1} \geq d(s, t)$, then PrefMatch $_{s}$ would have returned a cluster at a level lower than that of $C^{\prime}$ by Lemma 4.3.) Hence, we can apply the induction hypothesis to find a path from $v$ to $t$ of length at most $(1+\tau) d(v, t) \leq(1+\tau) 2 \eta_{i-\ell}$. The path from $s$ to $t$ as derived from Route $_{s}$ is of length at most $d(s, v)+(1+\tau) d(v, t)<d(s, t)+(1+\tau) 2 \eta_{i-\ell}$. The stretch of the path from $s$ is $t$ is then $1+(1+\tau) 2 \eta_{i-\ell} / d(s, t)$. This quantity is at most $1+\tau$ since $\tau \leq 1$ and we have chosen constants so that $\eta_{i-\ell} \leq \tau \varepsilon \rho^{i-1} / 4$.

\section{CONSTANT-DEGREE SPANNERS FOR DOUBLING METRICS}

Given a metric $(V, d)$ with doubling dimension $\alpha$ and $\tau>0$, this section shows how to construct a $(1+\tau)$-spanner whose maximum degree is bounded by $\left(2+\frac{1}{\tau}\right)^{O(\alpha)}$. We first recall the definition of spanners.

Definition $5.1((1+\tau)$-Spanner $)$. Let $(V, d)$ be a finite metric space. Suppose $G=$ $(V, E)$ is an undirected graph such that each edge $\{u, v\} \in E$ has weight $d(u, v)$, and $d_{G}(u, v)$ is the length of the shortest path between vertices $u$ and $v$ in $G$. The graph $G$, or equivalently, the set $E$ of edges, is a $(1+\tau)$-spanner for $(V, d)$ if for all pairs $u$ and $v$, $d_{G}(u, v) / d(u, v) \leq 1+\tau$.

Our construction consists of two phases. In the first phase described in Section 5.1, we construct a spanner $(V, \hat{E})$ from a nested sequence of nets $\left\{Y_{i}\right\}$; we include an edge if the endpoints are from the same net and "reasonably close" to each other. We then show that the edges in this spanner can be directed such that the out-degree of each vertex is bounded, and hence the spanner is sparse. We then have a second phase described in Section 5.2, in which we modify these edges in $\hat{E}$ to obtain another spanner, but now with bounded degree. Our main theorem is the following.

Theorem 5.2. Given a metric $(V, d)$ with doubling dimension $\alpha$, there exists $a(1+\tau)$ spanner such that the degree of every vertex is at most $\left(2+\frac{1}{\tau}\right)^{O(\alpha)}$.

\subsection{Basic Construction of Sparse $(1+\tau)$-Spanners for Doubling Metrics}

In this section, we show the existence of sparse spanners by giving an explicit construction. In particular, we have the following result.

Theorem 5.3. Given a metric $(V, d)$ with doubling dimension $\alpha$, there exists $a(1+\tau)$ spanner $\widehat{E}$ that has $\left(2+\frac{1}{\epsilon}\right)^{O(\alpha)} n$ edges.

The basic idea is to first construct a net-tree representing a sequence of nested nets of the metric space: this is fairly standard, and has been used earlier (e.g., in Talwar [2004] and Krauthgamer and Lee [2004]). A nearly linear-time construction of net trees is given by Har-Peled and Mendel [2005].

Net trees are formally defined in the following.

Definition 5.4 (Hierarchical Tree). A hierarchical tree for a set $V$ is a pair $(T, \varphi)$, where $T$ is a rooted tree, and $\varphi$ is a labeling function $\varphi: T \rightarrow V$ that labels each node of $T$ with an element in $V$, such that the following conditions hold:

1. Every leaf is at the same depth from the root.

2. The function $\varphi$ restricted to the leaves of $T$ is a bijection into $V$. 
3. If $u$ is an internal node of $T$, then there exists a child $v$ of $u$ such that $\varphi(v)=\varphi(u)$. This implies that the nodes mapped by $\varphi$ to any $x \in V$ form a connected subtree of $T$.

Definition 5.5 (Net Tree). A net tree for a metric $(V, d)$ is a hierarchical tree $(T, \varphi)$ for the set $V$ such that the following conditions hold:

1. Let $N_{i}$ be the set of nodes of $T$ that have height $i$. (The leaves have height 0 .) Suppose $\delta$ is the minimum pairwise distance in $(V, d)$. Let $0<r_{0}<\delta / 2$, and $r_{i+1}=2 r_{i}$, for $i \geq 0$. (Hence, $r_{i}=2^{i} r_{0}$.) Then, for $i \geq 0, \varphi\left(N_{i+1}\right)$ is an $r_{i+1}$-net for $\varphi\left(N_{i}\right)$.

2. Let node $u \in N_{i}$, and its parent node be $p_{u}$. Then, $d\left(\varphi(u), \varphi\left(p_{u}\right)\right) \leq r_{i+1}$.

In order to construct the spanner, we include an edge if the endpoints are from the same net in some scale and "reasonably close" to each other with respect to that scale. Using this idea, one can obtain the following theorem.

Theorem 5.6. Given a finite metric $M=(V, d)$ with doubling dimension bounded by $\alpha$, let $\tau>0$ and $(T, \varphi)$ be any net tree for $M$. For each $i \geq 0$, let

$$
E_{i}:=\left\{\{u, v\} \mid u, v \in \varphi\left(N_{i}\right), d(u, v) \leq\left(4+\frac{32}{\tau}\right) \cdot r_{i}\right\} \backslash E_{i-1}
$$

where $E_{-1}$ is the empty set. (Here the parameters $N_{i}, r_{i}$ are as in Definition 5.5.) Then $\widehat{E}:=\cup_{i} E_{i}$ forms a $(1+\tau)$-spanner for $(V, d)$, with the number of edges being $|\widehat{E}| \leq$ $\left(2+\frac{1}{\tau}\right)^{O(\alpha)}|V|$.

We prove Theorem 5.6 through Lemmas 5.7 and 5.10.

Lemma 5.7. The graph $(V, \widehat{E})$ is a $(1+\tau)$-spanner for $(V, d)$.

Proof. Let $\widehat{d}$ be the distance function induced by $(V, \widehat{E})$. Let $\gamma:=4+\frac{32}{\tau}$. We first show that each point in $V$ is close to some point in $\varphi\left(N_{i}\right)$ under the metric $\widehat{d}$.

ClaIm 5.8. For all $x \in V$, for all $i$, there exists $y \in \varphi\left(N_{i}\right)$ such that $\widehat{d}(x, y) \leq 2 r_{i}$.

Proof. We shall prove this by induction on $i$. For $i=0, \varphi\left(N_{0}\right)=V$. Hence, the result holds trivially.

Suppose $i \geq 1$. By the induction hypothesis, there exists $y^{\prime} \in \varphi\left(N_{i-1}\right)$ such that $\widehat{d}\left(x, y^{\prime}\right) \leq 2 r_{i-1}$. Since $\varphi\left(N_{i}\right)$ is an $r_{i}$-net of $\varphi\left(N_{i-1}\right)$, there exists $y \in \varphi\left(N_{i}\right) \subseteq \varphi\left(N_{i-1}\right)$ such that $d\left(y^{\prime}, y\right) \leq r_{i}=2 r_{i-1} \leq \gamma \cdot r_{i-1}$. Hence, $\left(y^{\prime}, y\right) \in \widehat{E}$ and $\widehat{d}\left(y^{\prime}, y\right)=d\left(y^{\prime}, y\right)$, which is at most $r_{i}$.

Finally, by the triangle inequality, $\widehat{d}(x, y) \leq \widehat{d}\left(x, y^{\prime}\right)+\widehat{d}\left(y^{\prime}, y\right) \leq 2 r_{i-1}+r_{i}=2 r_{i}$.

We next show that for any pair of vertices $x, y \in V, \widehat{d}(x, y) \leq(1+\tau) d(x, y)$. Suppose $r_{i} \leq d(x, y)<r_{i+1}$.

Suppose $q$ is the integer such that $\frac{8}{2^{q}} \leq \tau<\frac{16}{2^{q}}$, that is, $q:=\left\lceil\log _{2} \frac{8}{\tau}\right\rceil$.

We first consider the simple case when $i \leq q-1$. Then, $d(x, y)<2^{i+1} r_{0} \leq 2^{q} r_{0} \leq$ $\frac{16}{\tau} \cdot r_{0} \leq \gamma \cdot r_{0}$. Since $x, y \in \varphi\left(N_{0}\right)$, it follows that $(x, y) \in \widehat{E}$ and $\widehat{d}(x, y)=d(x, y)$.

Next we consider the case when $i \geq q$. Let $j:=i-q \geq 0$.

By Claim 5.8, there exist vertices $x^{\prime}, y^{\prime} \in \varphi\left(N_{j}\right)$ such that $\widehat{d}\left(x, x^{\prime}\right) \leq 2 r_{j}$ and $\widehat{d}\left(y, y^{\prime}\right) \leq 2 r_{j}$. 
We next show that $\left(x^{\prime}, y^{\prime}\right) \in \widehat{E}$. It suffices to show that $d\left(x^{\prime}, y^{\prime}\right) \leq \gamma \cdot r_{j}$.

$$
\begin{aligned}
d\left(x^{\prime}, y^{\prime}\right) & \leq d\left(x^{\prime}, x\right)+d(x, y)+d\left(y, y^{\prime}\right) & & \text { Triangle inequality) } \\
& \leq 2 r_{j}+r_{i+1}+2 r_{j} & & \text { (Choice of } \left.x^{\prime}, y^{\prime}, \text { and } i\right) \\
& =r_{j}\left(4+2 \cdot 2^{q}\right) & & (i=j+q) \\
& \leq r_{j}\left(4+\frac{32}{\tau}\right) & & \left(2^{q}<\frac{16}{\tau}\right) \\
& =\gamma \cdot r_{j} & &
\end{aligned}
$$

Hence, we have $\widehat{d}\left(x^{\prime}, y^{\prime}\right)=d\left(x^{\prime}, y^{\prime}\right)$. Note that by the triangle inequality,

$$
d\left(x^{\prime}, y^{\prime}\right) \leq d\left(x^{\prime}, x\right)+d(x, y)+d\left(y, y^{\prime}\right) \leq 4 \cdot r_{j}+d(x, y) .
$$

Finally, we obtain the desired upper bound for $\widehat{d}(x, y)$ :

$$
\begin{aligned}
\widehat{d}(x, y) & \leq \widehat{d}\left(x, x^{\prime}\right)+\widehat{d}\left(x^{\prime}, y^{\prime}\right)+\widehat{d}\left(y^{\prime}, y\right) & & \text { Triangle inequality) } \\
& \leq 8 \cdot r_{j}+d(x, y) & & \text { Choice of } x^{\prime}, y^{\prime}, \text { and (3)) } \\
& =\frac{8}{2^{q}} \cdot r_{i}+d(x, y) & & (j=i-q) \\
& \leq\left(1+\frac{8}{2^{q}}\right) d(x, y) & & \left(r_{i} \leq d(x, y)\right) \\
& \leq(1+\tau) d(x, y) & & \left(\frac{8}{2^{q}} \leq \tau\right)
\end{aligned}
$$

Observe that we have not used the definition of doubling dimension so far. We next proceed to show that the spanner $(V, \widehat{E})$ is sparse, by using the fact that the metric is doubling. We first show that for each vertex $u$, for each $i$, the number of edges in the $E_{i}$ incident on $u$ is small.

Claim 5.9. Define $\Gamma_{i}(u):=\left\{v \in V:\{u, v\} \in E_{i}\right\}$. Then, $\left|\Gamma_{i}(u)\right| \leq(4 \gamma)^{\alpha}$.

Proof. Observe that $\Gamma_{i}(u)$ is contained in a ball of radius at most $\gamma \cdot r_{i}$ centered at $u$. Moreover, since $S \subseteq \varphi\left(N_{i}\right)$, any two points in $S$ must be more than $r_{i}$ apart. Hence, from Proposition 2.1, it follows that $\left|\Gamma_{i}(u)\right| \leq(4 \gamma)^{\alpha}$.

Lemma 5.10. The number of edges in $\widehat{E}$ is at most $\left(2+\frac{1}{\tau}\right)^{O(\alpha)} n$.

PRoOF. It suffices to show that the edges of $\widehat{E}$ can be directed such that each vertex has out-degree bounded by $\left(2+\frac{1}{\tau}\right)^{O(\alpha)}$.

For each $v \in V$, define $i^{*}(v):=\max \left\{i \mid v \in \varphi\left(N_{i}\right)\right\}$. For each edge $(u, v) \in \widehat{E}$, we direct the edge from $u$ to $v$ if $i^{*}(u)<i^{*}(v)$. If $i^{*}(u)=i^{*}(v)$, the edge can be directed arbitrarily. By $\operatorname{arc}(u, v)$, we mean an edge that is directed from vertex $u$ to vertex $v$.

We now bound the out-degree of vertex $u$. Suppose there exists an $\operatorname{arc}(u, v) \in E_{i}$.

By definition of $E_{i}, d(u, v) \leq \gamma \cdot r_{i}$. Set $p=\left\lceil\log _{2} \gamma\right\rceil$. Hence, it is not possible for both $u$ and $v$ to be contained in $\varphi\left(N_{i+p}\right)$. Since $i^{*}(u) \leq i^{*}(v)$, it follows that $i^{*}(u) \leq i+p$. On the other hand, $u \in \varphi\left(N_{i}\right)$ and so $i^{*}(u) \geq i$. So, $i^{*}(u)-p \leq i \leq i^{*}(u)$.

There are at most $p+1=O(\log \gamma)$ values of $i$ such that $\bar{E}_{i}$ contains an edge directed out of $u$. By Claim 5.9, for each $i$, the number of edges in $E_{i}$ incident on $u$ is at most $(4 \gamma)^{\alpha}$.

Hence, the total number of edges in $\widehat{E}$ directed out of $u$ is $(4 \gamma)^{\alpha} \cdot O(\log \gamma)=(2+$ $\left.\frac{1}{\tau}\right)^{O(\alpha)}$.

Observe that in the proof of Lemma 5.7, we have actually shown that for any points $x$ and $y$, there is a short path of a particular form. This property will be useful when we construct spanners with a small hop diameter.

THEOREM 5.11. Consider the construction in Theorem 5.6. For any $x, y$ in $V$, the spanner $\widehat{E}$ contains a $(1+\tau)$-path of the following form. If $x_{0}$ and $y_{0}$ are the leaf nodes in $T$ with $\varphi\left(x_{0}\right)=x$ and $\varphi\left(y_{0}\right)=y$, and $x_{i}$ and $y_{i}$ are the ancestors of $x_{0}$ and $y_{0}$ at height 


\section{$i \geq 1$, then there exists $\bar{i}$ such that the path}

$$
x=\varphi\left(x_{0}\right), \varphi\left(x_{1}\right), \ldots, \varphi\left(x_{\bar{i}}\right), \varphi\left(y_{\bar{i}}\right), \ldots, \varphi\left(y_{1}\right), \varphi\left(y_{0}\right)=y
$$

is a $(1+\tau)$-path (after removing repeated vertices).

\subsection{Construction of $(1+\tau)$-Spanners with Bounded Degree}

We have shown that the edges in $\widehat{E}$ can be directed such that the out-degree of every vertex is bounded. We next describe how to modify $\widehat{E}$ to get another set of edges $\tilde{E}$ that has size at most that of $\widehat{E}$, but the resulting undirected graph $(V, \tilde{E})$ has bounded degree (Lemma 5.12). Moreover, we show in Lemma 5.13 that the modification preserves distances between vertices.

We form the new graph $(V, \tilde{E})$ by modifying the directed graph $(V, \widehat{E})$ in the following way.

Modification Procedure. Let $l$ be the smallest positive integer such that $\frac{1}{2^{l-1}} \leq \tau$. Then, $l=O\left(\log \frac{1}{\tau}\right)$.

For each $i$ and point $u$, define $M_{i}(u)$ to be the set of vertices $w$ such that $w \in \Gamma_{i}(u)$ and $(w, u)$ is directed into $u$ in $\widehat{E}$.

Let $I_{u}:=\left\{i \mid \exists v \in M_{i}(u)\right\}$. Suppose the elements of $I_{u}$ are listed in increasing order $i_{1}<i_{2}<\cdots$. To avoid double subscripts, we write $M_{j}^{u}:=M_{i_{j}}(u)$.

We next modify arcs going into each vertex $u$ in the following manner. For $1 \leq j \leq l$, we keep the arcs directed from $M_{j}^{u}$ to $u$. For $j>l$, we pick an arbitrary vertex $w \in M_{j-l}^{u}$ and, for each point $v \in M_{j}^{u}$, replace the $\operatorname{arc}(v, u)$ by the $\operatorname{arc}(v, w)$.

Observe that since $M_{j}^{u}$ is defined with respect to the directed graph $(V, \hat{E})$, the ordering of the $u$ s for which the modification is carried out is not important.

Let $(V, \tilde{E})$ be the resulting undirected graph. Since every edge in $\widehat{E}$ is either kept or replaced by another edge (which might be already in $\widehat{E}),|\tilde{E}| \leq|\widehat{E}|$.

LEMMA 5.12. Every vertex in $(V, \tilde{E})$ has degree bounded by $\left(2+\frac{1}{\tau}\right)^{O(\alpha)}$.

Proof. Let $\alpha$ be an upper bound for the out-degree of the graph $(V, \widehat{E})$. From Lemma 5.10, we have $\alpha=\left(2+\frac{1}{\tau}\right)^{O(\alpha)}$. Let $\beta$ be an upper bound for $\left|M_{i}(u)\right|$. We have $\beta \leq\left|\Gamma_{i}(u)\right|=\left(2+\frac{1}{\tau}\right)^{O(\alpha)}$.

We next bound the maximum degree of a vertex in $(V, \tilde{E})$. Consider a vertex $u \in V$. The edges incident on $u$ can be grouped as follows:

(1) There are at most $\alpha$ edges directed out of $u$ in $\widehat{E}$.

(2) Out of the edges in $\widehat{E}$ directed into $u$, at most $\beta l$ remain in $\tilde{E}$.

(3) New edges can be attached to $u$ in $(V, \tilde{E})$. For each arc $(u, v)$ directed out of $u$ in $\widehat{E}$, there can be at most $\beta$ new edges attaching to $u$ in $\tilde{E}$. The reason is $(u, v)$ can be in exactly one $E_{i}$ and so there exists unique $j$ such that $u \in M_{j}^{v}$. Hence, there could be potentially only at most $\left|M_{j+l}^{v}\right|$ new arcs directed into $u$ because of the arc $(u, v)$ in $\widehat{E}$.

Hence, the number of edges incident on $u$ in $(V, \tilde{E})$ is bounded by $\alpha+\beta l+\alpha \beta=$ $\left(2+\frac{1}{\tau}\right)^{O(\alpha)}$.

We next show that the modification from $(V, \widehat{E})$ to $(V, \tilde{E})$ does not increase the distance between any pair of vertices too much.

Lemma 5.13. Suppose $\tilde{d}$ is the metric induced by $(V, \tilde{E})$. Then, $\tilde{d} \leq(1+4 \tau) \widehat{d}$. 
Proof. It suffices to show that for each edge $(v, u) \in \widehat{E}$ removed, $\tilde{d}(v, u) \leq(1+$ $4 \tau) d(v, u)$.

Suppose $(v, u)$ in $\widehat{E}$ is directed into $u$. Then, by construction, $v \in M_{j}^{u}$ for some $j>l$.

Let $v_{0}=v$. Then, from our construction, for $0 \leq s \leq s_{j}:=\left\lfloor\frac{j-1}{l}\right\rfloor$, there exists $v_{s} \in M_{j-s l}^{u}$ such that for $0 \leq s<s_{j},\left(v_{s}, v_{s+1}\right) \in \tilde{E}$, and $\left(v_{s_{j}}, u\right) \in \tilde{E}$. Then, there is a path in $(V, \tilde{E})$ going from $v$ to $u$ traversing vertices in the following order: $v=v_{0}, v_{1}, \ldots, v_{s_{j}}, u$. By the triangle inequality, the quantity $\tilde{d}(v, u)$ is at most the length of this path, which we show is comparable to $d(v, u)$.

Claim 5.14. For $0 \leq s<s_{j}, d\left(u, v_{s+1}\right) \leq \tau d\left(u, v_{s}\right)$.

Proof. Note that $v_{s+1} \in M_{i}(u)$ and $v_{s} \in M_{j}(u)$ for some $i$ and $j$. From step 3 of our construction, $j-i \geq l$.

Since $d\left(v_{s}, u\right) \geq \gamma \cdot r_{j-1}$ and $d\left(v_{s+1}, u\right) \leq \gamma \cdot r_{i}$, it follows that $d\left(v_{s+1}, u\right) \leq \frac{2}{2^{l}} d\left(v_{s}, u\right) \leq$ $\tau d\left(v_{s}, u\right)$.

Claim 5.15. For $0 \leq s \leq s_{j}, d\left(v_{s}, u\right) \leq \tau^{s} d\left(v_{0}, u\right)$.

Proof. The claim can be proved by induction on $s$ and using Claim 5.14.

From the triangle inequality and Claims 5.14 and 5.15, we have

$$
d\left(v_{s}, v_{s+1}\right) \leq d\left(v_{s}, u\right)+d\left(u, v_{s+1}\right) \leq(1+\tau) d\left(v_{s}, u\right) \leq(1+\tau) \tau^{s} d\left(v_{0}, u\right) .
$$

Finally, we have

$$
\begin{aligned}
\tilde{d}(v, u) & \leq \sum_{s=0}^{s_{j}-1} d\left(v_{s}, v_{s+1}\right)+d\left(v_{s_{j}}, u\right) \quad \text { (Triangle inequality) } \\
& \leq \sum_{s=0}^{s_{j}-1}(1+\tau) \tau^{s} d\left(v_{0}, u\right)+\tau^{s_{j}} d\left(v_{0}, u\right) \quad((4) \text { and Claim 5.15) } \\
& \leq \frac{1+\tau}{1-\tau} d\left(v_{0}, u\right) \\
& \leq(1+4 \tau) d(v, u)
\end{aligned}
$$

The last inequality follows from the fact that for $0<\tau<\frac{1}{2}, \frac{1+\tau}{1-\tau} \leq 1+4 \tau$.

Finally, we show that $(V, \tilde{E})$ is the desired spanner.

TheOREm 5.16. Given a metric $(V, d)$ with doubling dimension $\alpha$, there exists $a(1+\tau)$ spanner such that the degree of every vertex is at most $\left(2+\frac{1}{\tau}\right)^{O(\alpha)}$.

Proof. We show that $\tilde{E}$ gives the desired spanner. Lemma 5.12 gives the bound on its degree. From Lemmas 5.7 and 5.13, we have $\tilde{d} \leq(1+4 \tau) \widehat{d} \leq(1+4 \tau)(1+\tau) d \leq(1+7 \tau) d$, for $0<\tau \leq \frac{1}{2}$. Substituting $\tau:=\frac{\tau^{\prime}}{7}$ gives the required result.

\section{ACKNOWLEDGMENTS}

We would like to thank the anonymous referees for their comments on improving the presentation of the article.

\section{REFERENCES}

Ittai Abraham, Yair Bartal, and Ofer Neiman. 2007. Local embeddings of metric spaces. In Proceedings of the 39th Annual ACM Symposium on Theory of Computing (STOC'07). 631-640. DOI:http://dx.doi. org/10.1145/1250790.1250883

Ittai Abraham, Cyril Gavoille, Andrew Goldberg, and Dahlia Malkhi. 2006. Routing in networks with low doubling dimension. In Proceedings of the 26th International Conference on Distributed Computing Systems (ICDCS'06). 75. 
Noga Alon and Joel Spencer. 1992. The Probabilistic Method. Wiley Interscience, New York.

Sunil Arya, Gautam Das, David M. Mount, Jeffrey S. Salowe, and Michiel H. M. Smid. 1995. Euclidean spanners: Short, thin, and lanky. In Proceedings of the Twenty-Seventh Annual ACM Symposium on Theory of Computing (STOC'95). 489-498.

Baruch Awerbuch, Amotz Bar-Noy, Nathan Linial, and David Peleg. 1990. Improved routing strategies with succinct tables. J. Algorithms 11, 3 (1990), 307-341.

Baruch Awerbuch and David Peleg. 1990. Sparse partitions (extended abstract). In FOCS. 503-513.

Baruch Awerbuch and David Peleg. 1992. Routing with polynomial communication-space trade-off. SIAM J. Discrete Math. 5, 2 (1992), 151-162.

Yair Bartal. 1996. Probabilistic approximations of metric spaces and its algorithmic applications. In Proceedings of the 37th Symposium on the Foundations of Computer Science (FOCS'96). 184-193.

József Beck. 1991. An algorithmic approach to the Lovász local Lemma. I. Random Structures Algorithms 2 , 4 (1991), 343-365.

Hubert T.-H. Chan and Anupam Gupta. 2006. Small hop-diameter sparse spanners for doubling metrics. In Proceedings of the Seventeenth Annual ACM-SIAM Symposium on Discrete Algorithm (SODA'06). 70-78.

T.-H. Hubert Chan, Anupam Gupta, and Kunal Talwar. 2010. Ultra-low-dimensional embeddings for doubling metrics. J. ACM 57, 4 (2010). DOI : http://dx.doi.org/10.1145/1734213.1734215

T.-H. Hubert Chan, Mingfei Li, Li Ning, and Shay Solomon. 2015. New doubling spanners: Better and simpler. SIAM J. Comput. 44, 1 (2015), 37-53. DOI : http://dx.doi.org/10.1137/130930984

Lenore J. Cowen. 2001. Compact routing with minimum stretch. J. Algorithms 38, 1 (2001), 170-183. 10th Annual ACM-SIAM Symposium on Discrete Algorithms (1999).

Gautam Das, Giri Narasimhan, and Jeffrey Salowe. 1995. A new way to weigh malnourished Euclidean graphs. In Proceedings of the 6th Annual ACM-SIAM Symposium on Discrete Algorithms (SODA'95). ACM, New York, 215-222.

Michel Marie Deza and Monique Laurent. 1997. Geometry of Cuts and Metrics. Algorithms and Combinatorics, Vol. 15. Springer-Verlag, Berlin. xii+587 pages.

Michael Elkin and Shay Solomon. 2013. Optimal Euclidean spanners: Really short, thin and lanky. In Symposium on Theory of Computing Conference (STOC'13). 645-654. DOI:http://dx.doi.org/10. 1145/2488608.2488691

Greg N. Frederickson and Ravi Janardan. 1988. Designing networks with compact routing tables. Algorithmica 3 (1988), 171-190.

Greg N. Frederickson and Ravi Janardan. 1989. Efficient message routing in planar networks. SIAM J. Comput. 18, 4 (1989), 843-857.

Jie Gao, Leonidas J. Guibas, and An Nguyen. 2004. Deformable spanners and applications. In Proceedings of the 20th ACM Symposium on Computational Geometry (SCG'04). 190-199. DOI :http://dx.doi.org/10. $1145 / 997817.997848$

Cyril Gavoille. 2001. Routing in distributed networks: Overview and open problems. ACM SIGACT News Distributed Computing Column 32, 1 (March 2001), 36-52.

Cyril Gavoille and Marc Gengler. 2001. Space-efficiency for routing schemes of stretch factor three. J. Parallel Distrib. Comput. 61, 5 (2001), 679-687. DOI : http://dx.doi.org/10.1006/jpdc.2000.1705

Lee-Ad Gottlieb and Liam Roditty. 2008a. Improved algorithms for fully dynamic geometric spanners and geometric routing. In Proceedings of the Nineteenth Annual ACM-SIAM Symposium on Discrete Algorithms (SODA'08). 591-600.

Lee-Ad Gottlieb and Liam Roditty. 2008b. An optimal dynamic spanner for doubling metric spaces. In Proceedings of the 16th Annual European Symposium on Algorithms (ESA'08). 478-489.

Anupam Gupta, Robert Krauthgamer, and James R. Lee. 2003. Bounded geometries, fractals, and lowdistortion embeddings. In Proceedings of the 44th Symposium on the Foundations of Computer Science (FOCS'03). 534-543.

Sariel Har-Peled and Manor Mendel. 2005. Fast constructions of nets in low dimensional metrics, and their applications. In Proceedings of the 21st Symposium on Computational Geometry (SOCG'05). 150-158.

Juha Heinonen. 2001. Lectures on Analysis on Metric Spaces. Springer-Verlag, New York.

Kirsten Hildrum, John D. Kubiatowicz, Satish Rao, and Ben Y. Zhao. 2002. Distributed object location in a dynamic network. In Proceedings of the 14th Annual ACM symposium on Parallel Algorithms and Architectures (SPAA'02). 41-52.

David R. Karger and Matthias Ruhl. 2002. Finding nearest neighbors in growth-restricted metrics. In Proceedings of the 34th ACM Symposium on the Theory of Computing (STOC'02). 63-66. citeseer. nj.nec.com/556595.html. 
Leonard Kleinrock and Farouk Kamoun. 1977. Hierarchical routing for large networks. Performance evaluation and optimization. Comput. Netw. 1, 3 (1977), 155-174.

Goran Konjevod, Andrea W. Richa, and Donglin Xia. 2007. Optimal scale-free compact routing schemes in doubling networks. In Proceedings of the 18th ACM-SIAM Symposium on Discrete Algorithms (SODA'07). 939-948.

Robert Krauthgamer and James R. Lee. 2003. The intrinsic dimensionality of graphs. In Proceedings of the 35th ACM Symposium on the Theory of Computing (STOC'03). ACM Press, 438-447. DOI : http://dx.doi.org/10.1145/780542.780607

Robert Krauthgamer and James R. Lee. 2004. Navigating nets: Simple algorithms for proximity search. In Proceedings of the 15th ACM-SIAM Symposium on Discrete Algorithms (SODA'04). 798-807.

Robin A. Moser and Gábor Tardos. 2010. A constructive proof of the general Lovász local lemma. J. ACM 57, 2 (2010). DOI : http://dx.doi.org/10.1145/1667053.1667060

David Peleg. 2000. Distributed Computing: A Locality-Sensitive Approach. Discrete Mathematics and Applications. Society for Industrial and Applied Mathematics (SIAM), Philadelphia, PA. xvi + 324 pages.

David Peleg and Eli Upfal. 1989. A trade-off between space and efficiency for routing tables. J. Assoc. Comput. Mach. 36, 3 (1989), 510-530.

C. Greg Plaxton, Rajmohan Rajaraman, and Andréa W. Richa. 1999. Accessing nearby copies of replicated objects in a distributed environment. Theory Comput. Syst. 32, 3 (1999), 241-280. ACM Symposium on Parallel Algorithms and Architectures (Padua, 1996).

Rajmohan Rajaraman, Andrea W. Richa, Berthold Vocking, and Gayathri Vuppuluri. 2001. A data tracking scheme for general networks. In Proceedings of the 13th Annual ACM Symposium on Parallel Algorithms and Architectures (SPAA'01). ACM Press, 247-254. DOI : http://dx.doi.org/10.1145/378580.378670

Jeffrey S. Salowe. 1992. On Euclidean spanner graphs with small degree. In Proceedings of the 8th Annual Symposium on Computational Geometry (SCG'92). 186-191. DOI:http://dx.doi.org/10.1145/ 142675.142716

Aleksandrs Slivkins. 2005. Distance estimation and object location via rings of neighbors. In Proceedings of the 24th Annual ACM Symposium on Principles of Distributed Computing (PODC'05). 41-50.

Shay Solomon. 2014. From hierarchical partitions to hierarchical covers: Optimal fault-tolerant spanners for doubling metrics. In Symposium on Theory of Computing (STOC'14). 363-372. DOI:http://dx.doi. org/10.1145/2591796.2591864

Kunal Talwar. 2004. Bypassing the embedding: Algorithms for low-dimensional metrics. In Proceedings of the 36th ACM Symposium on the Theory of Computing (STOC'04). 281-290.

Pravin M. Vaidya. 1991. A sparse graph almost as good as the complete graph on points in K dimensions. Discrete Comput. Geom. 6 (1991), 369-381. DOI:http://dx.doi.org/10.1007/BF02574695

Received November 2012; revised April 2015; accepted April 2016 